\title{
过渡金属催化氮原子导向的芳基邻位 $\mathrm{C}$ - $\mathrm{H}$ 键硼化反应研究进展
}

\author{
罗欢欢裴 娜张 敬* \\ (武汉大学高等研究院 武汉 430000)
}

\begin{abstract}
摘要 芳基嗍化合物在合成化学、材料化学和生物医学领域都有着广泛的应用, 其合成方法一直是有机合成领域中的 研究热点. 导向基团辅助过渡金属催化的 $\mathrm{C}-\mathrm{H}$ 键嗍化反应具有步骤经济性, 底物多样性, 高区域选择性的优点. 其中 含氮原子导向基团底物的嗍化反应引起了学者们的兴趣, 因为 $\mathrm{N}, \mathrm{C}$ 鳌合的四配位有机嗍化物是重要的光电材料. 按照 不同过渡金属(铱、错、钯、钓)总结了近年来含氮原子导向的芳香化合物邻位 $\mathrm{C}-\mathrm{H}$ 键硼化反应的进展.

关键词 邻位 $\mathrm{C}-\mathrm{H}$ 键活化; 嗍化; 氮原子导向; 过渡金属催化
\end{abstract}

\section{Advances in Nitrogen-Directed Aromatic Compound ortho- $\mathrm{C}-\mathrm{H}$ Bond Borylation Catalyzed by Transition Metals}

\author{
Luo, Huanhuan Pei, Na Zhang, Jing* \\ (Institute for Advanced Studies, Wuhan University, Wuhan 430000)
}

\begin{abstract}
Aromatic boron compounds have been widely used in synthetic chemistry, materials and medicinals, and developing new methods for their synthesis has been a hot topic. Directing groups assisted $\mathrm{C}-\mathrm{H}$ bond borylation catalyzed by transition metal has significant advantages in step-economy, substrates diversity and high regio-selectivity. The borylation of nitrogen-based substrates has attracted interest from researchers, because four-coordinated organoboron compounds chelated by N,C are important photoelectric materials. In this paper, the preparation of aromatic boron compounds from ortho- $\mathrm{C}-\mathrm{H}$ bond borylation catalyzed by $\mathrm{Ir}, \mathrm{Rh}, \mathrm{Ru}, \mathrm{Pd}$ assisting by directing groups containing nitrogen atoms is summarized.
\end{abstract}

Keywords ortho-C $-\mathrm{H}$ activation; borylation; nitrogen-directed; transition metal catalyze

有机嗍试剂在诸多领域都扮演着非常重要的角色. 例如，在有机合成化学中，良好的稳定性与反应活性使 得其在复杂分子构造和药物合成领域中被广泛应用 ${ }^{[1-4]}$. 在光电材料中, 有机嗍化合物通常含有易修饰的 $\pi$ 共轭 结构, 使其具备发光性强, 载流子迁移率较高的特点, 被广泛用于有机光电材料的发光材料和电子转移材 料 ${ }^{[-6]}$. 除此之外, 在生物医学 ${ }^{[7]}$ 领域中的应用也逐渐被 人们所开发.

芳基硼酸及其衍生物的主要合成方法是芳基卤化 物的嗍化反应, 分为两类方法: 第一种是传统方法, 先 将芳基 $\mathrm{C}-\mathrm{X}$ 键 $(\mathrm{X}=\mathrm{Cl}, \mathrm{Br}, \mathrm{I})$ 进行金属化，制备得到有 机金属试剂, 如芳基格氏试剂与芳基锂试剂[8-9], 然后再 与硼试剂反应得到芳基硼化合物. 该策略具有试剂成本 低的优点, 但是主要存在以下两点不足: (1)反应需要预 制备芳基金属试剂, 增加了步骤的繁琐性; (2)反应体系
官能团兼容性较差. 第二种是过渡金属(主要是金属钯) 催化的卤代芳烃与硼试剂的偶联反应，直接得到相应的 芳基嗍酸化合物 ${ }^{[10]}$. 该方法相较于前一种具有反应条 件更加温和, 官能团兼容性好, 步骤经济的特点. 但是 该方法需要过渡金属催化剂, 除了增加成本, 也带来了 金属残留的问题. 所用的芳基卤主要是芳基溴或者芳基 碘试剂, 近期对于相对廉价的氯代芳烃的硼化反应的研 究也取得了一定的进展 ${ }^{[1-12]}$.

近年来, $\mathrm{C}-\mathrm{H}$ 键官能化受到人们的广泛关注，该策 略不仅提高了反应的原子与步骤经济性, 并且进一步拓 宽了底物范围, 为构建新的碳碳键或碳杂键提供了更直 接的方法. 在 $\mathrm{C}-\mathrm{H}$ 键向 $\mathrm{C}-\mathrm{B}$ 键转化的发展中 ${ }^{[13-14]}$, 过 渡金属催化 ${ }^{[15-19]}$ 策略因其良好的催化活性成为实现该 转化的主要方法. 1993 年, Marder 课题组 ${ }^{[20]}$ 在使用甲苯 为溶剂制备三嗍基铱络合物时, 通过气相色谱/质谱

* Corresponding author. E-mail: jzhangwhu@whu.edu.cn

Received March 6, 2021; revised April 2, 2021; published online May 7, 2021.

Project supported by the Fundamental Research Funds for the Central Universities (No. 2042019kf0008).

中央高校基本科研业务费专项资金(No. 2042019kf0008)资助项目. 
(GC/MS) 首次检测到反应中少量甲苯嗍基化产物的存 在. 1995 年, Hartwig 等 ${ }^{[21]}$ 通过紫外光照射金属嗍络合物 $(\mathrm{M}=\mathrm{Re}, \mathrm{Fe}, \mathrm{Mn})$ 的方法, 首次以较高收率得到了芳香化 合物和烯烃的硼化产物. 在一系列过渡金属催化 $\mathrm{C}-\mathrm{H}$ 键硼化的研究工作中, 反应的选择性主要来源于底物的 位阻效应和电子效应 ${ }^{[15,22]}$, 如何实现高区域选择性成为 该领域的主要挑战. 导向基团的引入可以诱导金属对特 定位点的 $\mathrm{C}-\mathrm{H}$ 键进行活化, 这也成为高选择性 $\mathrm{C}-\mathrm{H}$ 键嗍化反应的补充策略 ${ }^{[23-28]} .2008$ 年, Hartwig 课题组 ${ }^{[29]}$ 使用氢硅烷作为无痕导向基团, 实现了铱催化苯酚和苯 胺衍生物的邻位 $\mathrm{C}-\mathrm{H}$ 键硼化反应, 为导向基团辅助的 $\mathrm{C}-\mathrm{H}$ 键硼化提供了可能.

目前, 在导向的 $\mathrm{C}-\mathrm{H}$ 键嗍化反应中涉及到导向基

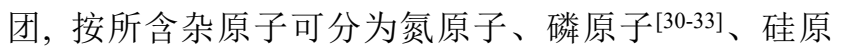
子[34-35]、氧原子 ${ }^{[36-37]}$ 等. 其中氮原子导向的 $\mathrm{C}-\mathrm{H}$ 键嗍 化反应产物, 由于 $\mathrm{N}-\mathrm{B}$ 键的路易斯酸碱对相互作用成 了 $\pi$ 共轭结构, 使得此类产物具有独特的光学特性, 在 有机光电材料[38-40]、太阳能电池 ${ }^{[41-42]}$ 等领域有潜在的应 用价值. 对于目前有关过渡金属催化的含氮原子导向基 团参与的芳香化合物的 $\mathrm{C}-\mathrm{H}$ 键硼化反应还没有系统的 总结, 本文以反应所用不同过渡金属(铱、铑、钉、钯) 进行分类综述, 其中铱催化的硼化反应相对比较成熟, 为便于读者的理解, 我们按照不同类型的配体进行总 结.

\section{1 铱催化 C- $\mathrm{H}$ 键磞化反应}

1999 年, Smith 等 ${ }^{[43]}$ 使用硼基铱络合物催化 $\mathrm{C}-\mathrm{H}$ 键活化得到了苯硼酸频哪醇酯. 此后, Ishiyama、Miyaura 和 Hartwig 等 ${ }^{[4-45]}$ 对配体及铱催化剂进行一系列篮选后 得出, $[\operatorname{Ir}(\mu-\mathrm{X})(\operatorname{cod})]_{2} / \mathrm{d} t$ bpy (dtbpy: 二叔丁基联吡啶; $=\mathrm{Cl}, \mathrm{OMe} ; \operatorname{cod}=1,5$-环辛二烯) 是 $\mathrm{C}-\mathrm{H}$ 键硼化的有效 催化体系, 反应的关键是形成了 16 电子的三硼基铱络 合物 $\mathbf{A}$ (Scheme 1). 该体系的高催化活性促使底物范围 进一步扩大, 然而 $[\operatorname{Ir}(\mu-\mathrm{X})(\operatorname{cod})]_{2} / \mathrm{d} \mathrm{tbpy}$ 催化体系中的双 齿氮配体使催化剂中心没有多余的空位留给含强配位 原子导向基团的底物(例如氮原子), 使此类底物的 $\mathrm{C}-$ $\mathrm{H}$ 键活化受限. 获得氮原子导向的区域选择性 $\mathrm{C}-\mathrm{H}$ 键 硼化产物, 主要是通过配体调控产生额外的空位的方 法, 例如单齿配体和介稳双齿配体的使用. 其次是利用 底物中的氮原子与催化剂上的配体形成非共价作用实 现导向，避免了导向基团与金属中心的直接配位.

\section{1 单齿配体}

使用单齿配体代替双齿配体, 即可产生新的空位来 促使 $\mathrm{C}-\mathrm{H}$ 键活化. Ishiyama 和 Miyaura 等 ${ }^{[46-47]}$ 使用单齿 膦配体 $\left[3,5-\left(\mathrm{CF}_{3}\right)_{2} \mathrm{C}_{6} \mathrm{H}_{3}\right]_{3} \mathrm{P}$ 和 $\left(\mathrm{C}_{6} \mathrm{H}_{5}\right)_{3} \mathrm{As}$ 实现了苯甲酸酯
的邻位 $\mathrm{C}-\mathrm{H}$ 键硼化反应. 随后, Ishiyama 和 Ito 等 ${ }^{[48]}$ 将 该体系应用到含氮原子导向的嗍化反应中(Eq. 1). 在对 反应条件进行进一步优化后, 得出使用三(五氟苯基)膦 为配体可以与联嗍酸频哪醇酯 $\left(\mathrm{B}_{2} \mathrm{pin}_{2}\right)$ 反应实现醛亚胺 导向的邻位 $\mathrm{C}-\mathrm{H}$ 键嗍化反应. 降冰片烯的加入是为了 捕获反应中产生的氢气，从而抑制还原产物的生成，反 应中并未检测到双硼化的副产物. 但是为了保证反应的 产率, 芳香醛亚胺衍生物需过量.

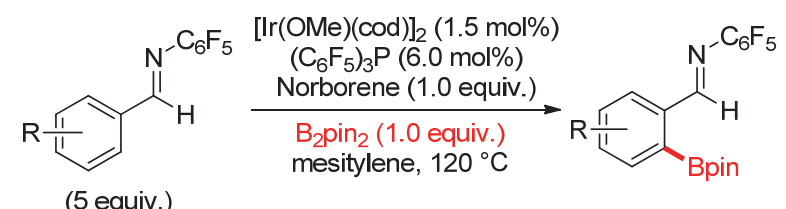

\section{2 双齿配体}

2011 年, Lassaletta 和 Fernández 等 ${ }^{[49]}$ 在经过一系列 的研究发现, 使用 $0.5 \mathrm{~mol} \%$ 的 $[\operatorname{Ir}(\mathrm{OMe})(\mathrm{cod})]_{2}$, 介稳 $N, N$-双齿配体 $\mathbf{L 1}$, 在催化量 (5 $\mathrm{mol} \%)$ 的频哪醇硼烷 (HBpin)的共催化下, 可以和 $\mathrm{B}_{2} \mathrm{pin}_{2}$ 获得 2-芳基吡啶(异 喹啉)及 $N, N$-二甲基腙类化合物的邻位嗍化产物(Eq. 2). 其中吡啶腙结构的介稳双齿配体是实现反应高选择性 的关键. 该体系可适用一系列带不同电性的底物，吡啶 环上带给电子官能团会增加导向基团的配位能力, 所以 反应更倾向于此类底物. 对于少数位阻较小的 2 -芳基吡 啶类底物检测到双硼化的副产物. 通过 ${ }^{11} \mathrm{~B}$ NMR 的低 位移和 $\mathrm{X}$ 射线结果，确定了产物中 $\mathrm{B}-\mathrm{N}$ 键的相互作用， 而对于位阻效应大的底物, 则不存在此作用. 这种非共 价相互作用使产物更趋向于平面, 增强了化合物的发光 性质，但也限制了底物以高产率获得双官能团化产物， 因为破坏这种作用需要更高的能量. 根据之前的设想, 推测反应的机理可能是(Scheme 1): 底物上的氮原子与 金属发生配位的同时，介稳配体上弱配位的氮发生解 离, 留出一个空位促进邻位的 $\mathrm{C}-\mathrm{H}$ 键活化得到中间体

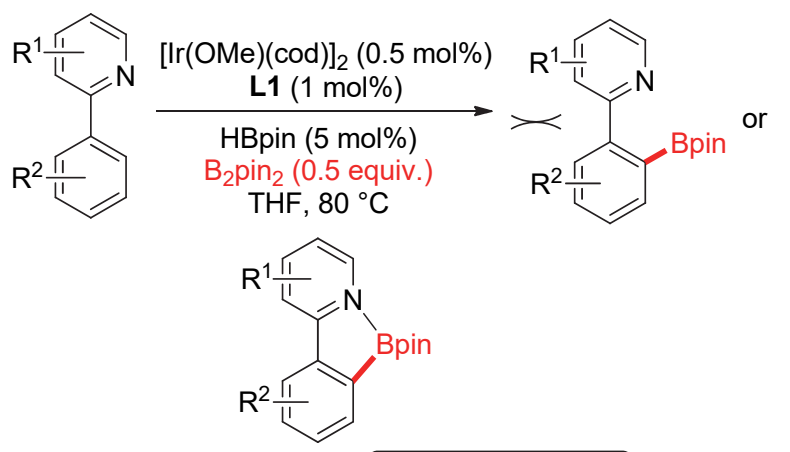




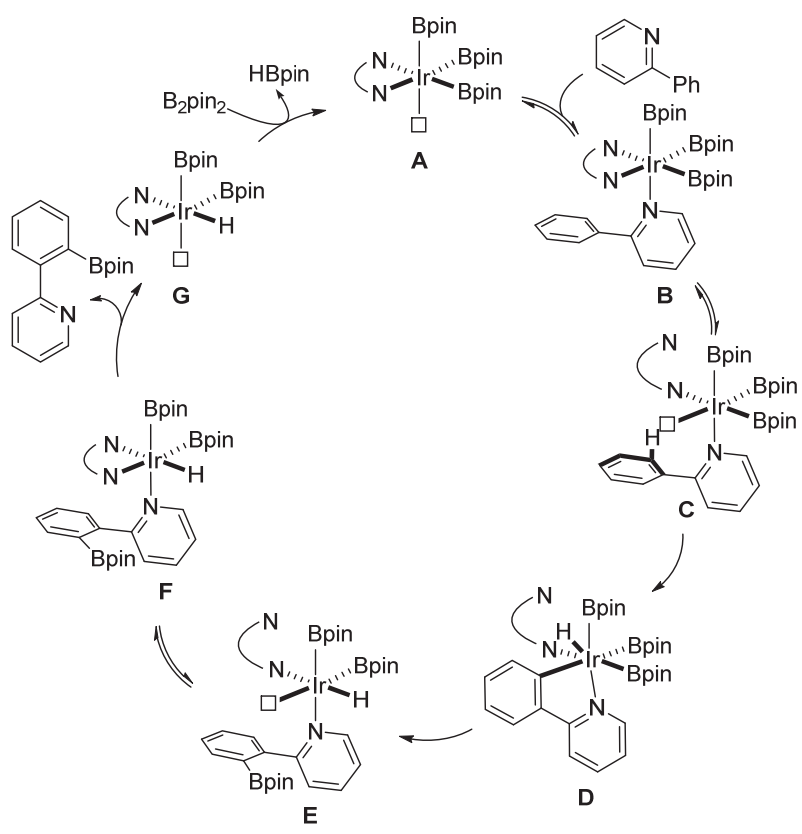

图式 1 介稳配体参与铱催化 2-芳基吡啶的 $\mathrm{C}-\mathrm{H}$ 键嗍化的机 理

Scheme 1 Mechanism of $\mathrm{C}-\mathrm{H}$ bond borylation of 2-arylpyridine catalyzed by iridium with hemilabile ligand

$\mathbf{D}$, 中间体 $\mathbf{D}$ 经过还原消除得到产物和 $\operatorname{Ir}(\mathrm{Bpin})_{2}(\mathrm{H})$ 物种 $\mathbf{G}$, 该物种与 $\mathrm{B}_{2} \mathrm{pin}_{2}$ 转金属化再生催化剂中间体 $\mathbf{A}$.

$N, N$ 二 二甲基腙导向的邻位单硼化产物不存在 $\mathrm{N}-\mathrm{B}$ 键相互作用，因为腙上的两个甲基与 Bpin 上的甲基存 在一定的位阻效应. 此外, 嗍化产物的 ${ }^{1} \mathrm{H}$ NMR 的去屏 蔽效应以及单晶衍射的结果证实了氢键的存在. 就表明 腙作为导向基团可以活化另一个邻位 $\mathrm{C}-\mathrm{H}$ 键, 促进双 嗍化反应. Lassaletta 和 Fernández 等 ${ }^{[50]}$ 在上述催化体系 的基础上, 增加催化剂和 $\mathrm{B}_{2} \mathrm{pin}_{2}$ 的量, 并适当延长反应 时间, 选择性地得到了双硼化的产物(Scheme 2). 反应 可兼容不同电性的官能团, 包括具有一定配位能力的氰 基. 对于间位取代的或多取代的底物, 由于位阻影响会 在一定程度上降低反应的速率，通过延长反应时间，可 以促进反应的完全转化. 此类产物可作为反应中间体, 结合 Suzuki-Miyaura 反应或氧化反应, 实现连续功能化, 得到不对称的芳烃化合物.

2012 年, Lassaletta、Fernández 和 Ros 等 ${ }^{[51]}$ 对介稳配 体进行修饰, 于 $\mathbf{L} 1$ 的吡啶上增加了 $N, N$-二甲基(Scheme $3)$, 起到了稳定催化剂中间体的同时促进反应中弱配位 氮的解离作用. 他们使用 $[\operatorname{Ir}(\mathrm{OMe})(\mathrm{cod})]_{2}$ 为催化剂, $\mathbf{L 2}$ 为配体, 实现了 $N, N$-二甲基腙与 HBpin 的邻位单硼化反 应, 反应中检测到少量双硼化的副产物. 结合 SuzukiMiyaura 交叉偶联，一锅法得到 $N, N$-二甲基腙类衍生物 邻位芳基化产物.

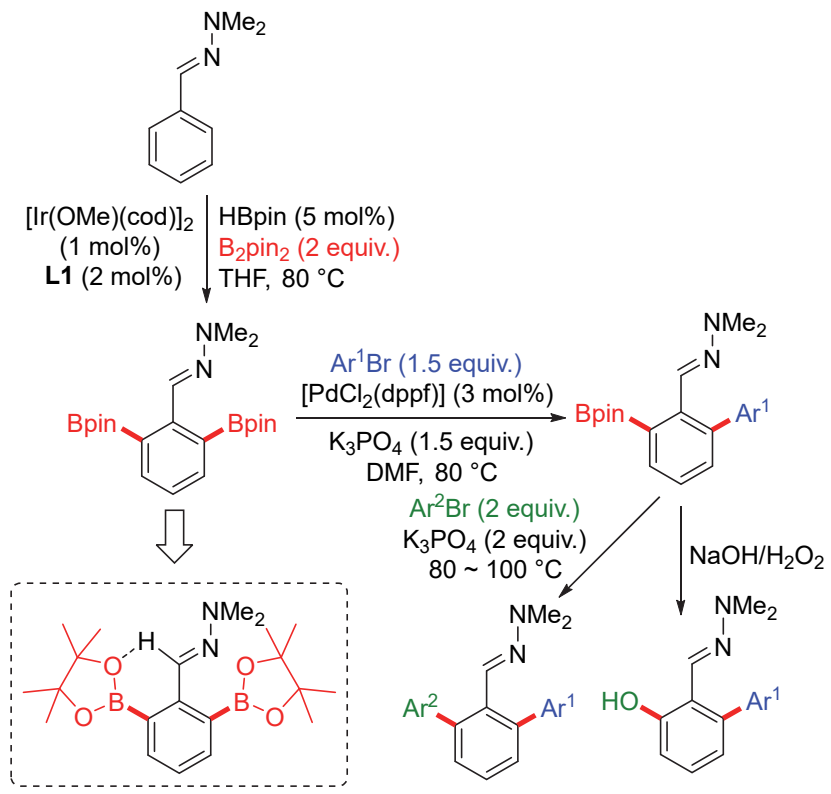

图式 2 铱催化 $N, N$-二甲基腙类衍生物双嗍化反应

Scheme 2 Iridium catalyzed diborylation of $N, N$-dimethylhydrazones derivates
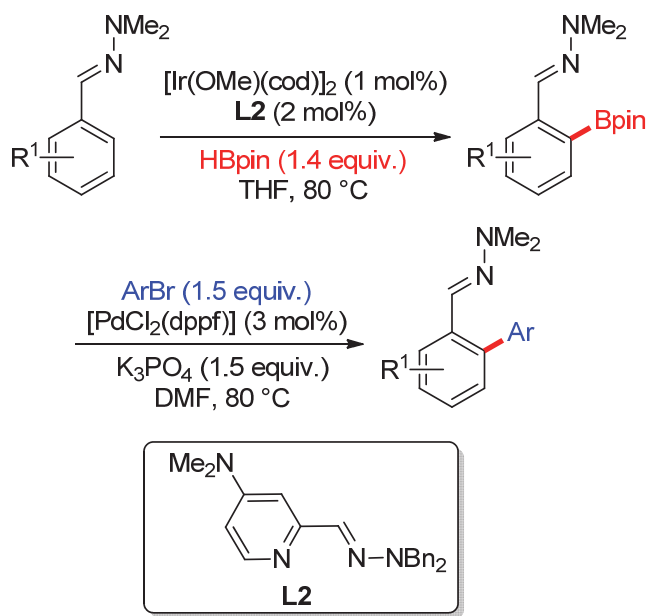

图式 3 铱催化 $N, N$-二甲基腙类衍生物单嗍化反应

Scheme 3 Iridium catalyzed monoborylation of $N, N$-dimethylhydrazones derivates

同年, Clark 课题组 ${ }^{[52]}$ 使用氨甲基吡啶作为配体，实 现了 $N, N$-二甲基茮胺的邻位硼化反应(Scheme 4). 作者 还尝试了含其他类型的导向基团的底物, 例如 $N, N-$ 二甲 基-2-苯基乙胺、 $N, N$-二甲基-1-苯基乙胺以及咪唑类衍生 物均适用，但当使用酰胺作为导向基团时，反应并没有 检测到相应的产物. 为验证反应的机理，作者换用配体 L4, 仍能以 $91 \%$ 的转化率得到单双取代的混合产物. 说 明该反应不是通过氢键导向的机理，而是与 Lassaletta 所使用介稳配体的策略相似, 通过配体上弱配位氮的解 离产生可供 $\mathrm{C}-\mathrm{H}$ 键活化的空位. 该体系也可以与 Suzuki 偶联反应串联，一锅两步法实现 $N, N$-二甲基茮胺 
的邻位芳基化反应 ${ }^{[53]}$. 2015 年，该课题组 ${ }^{[54]}$ 进一步研究 了此类双齿配体在硼化反应中的影响, 发现配体的位阻 效应和电子效应均会影响反应的转化率和选择性, 其中 双齿配体 L5 的更小咬角是倾向于形成单取代产物和进 一步提升反应转化率的关键因素.
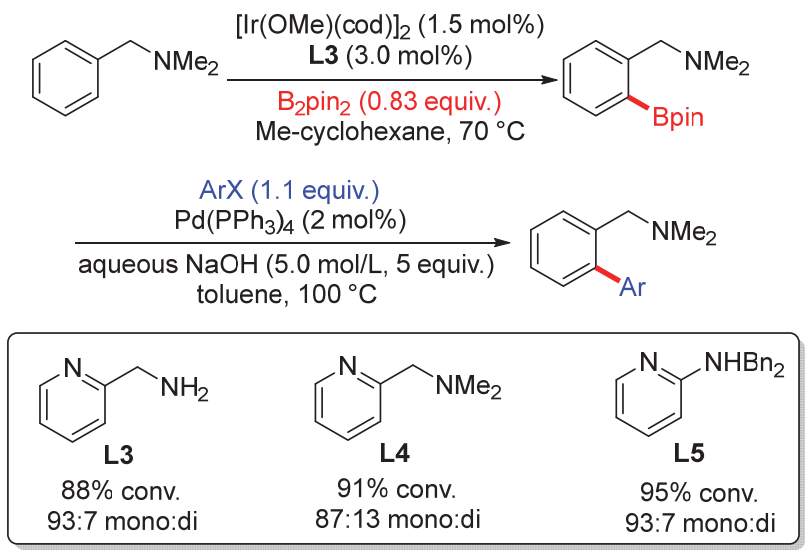

图式 4 铱催化 $N, N$-二甲基苄胺类衍生物的硼化反应 Scheme 4 Iridium catalyzed borylation of $N, N$-dimethylbenzylamine derivates

2012 年, Smith、Maleczka 和 Singleton 等 ${ }^{[55]}$ 在 $[\operatorname{Ir}(\mu-$ $\mathrm{X})(\mathrm{cod})]_{2} / \mathrm{d} t \mathrm{bpy}$ 催化体系的基础上, 通过苯胺底物上的 $\mathrm{NH}$ 与 16 电子活性中间体 $\operatorname{Ir}(\text { Bpin })_{3}$ 上的氧原子形成氢键 相互作用的策略, 实现了球外导向的 $\mathrm{C}-\mathrm{H}$ 键硼化反应 (Scheme 5). 作者使用叔丁氧羰基(Boc)保护的苯胺作为 芳香底物, 实现了与 $B_{2} p_{2} n_{2}$ 的邻位硼化反应. 值得注意 的是, 当胺上的两个氢均被 Boc 取代时, 反应更倾向于 发生在间位. 这种利用导向基团与配体相互作用的策 略, 为实现其他可形成氢键的原子作为导向基团的嗍化 反应提供了可能.

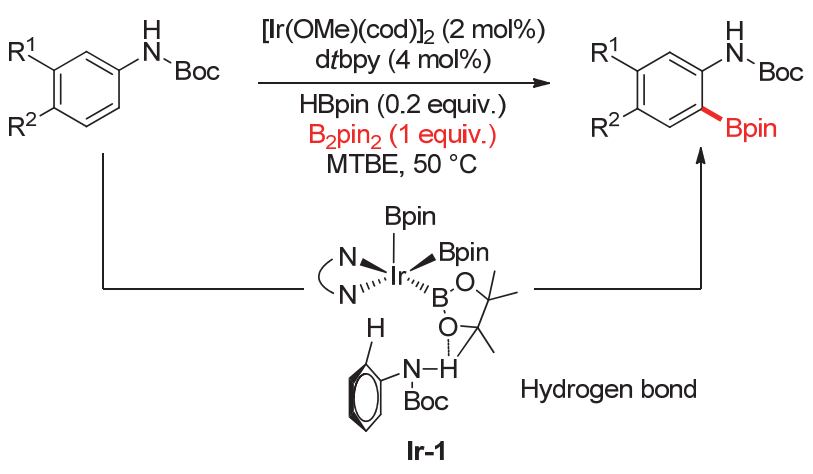

图式 5 铱催化球外导向的 $\mathrm{C}-\mathrm{H}$ 键硼化反应

Scheme 5 Iridium catalyzed outer-sphere directed $\mathrm{C}-\mathrm{H}$ bond borylation

2016 年, Chattopadhyay 等[56]发展了铱催化苯甲醛 类衍生物的 $\mathrm{C}-\mathrm{H}$ 键硼化反应. 他们先将芳基醛预制备 成亚胺类化合物, 再通过调控不同配体, 选择性地得到
了邻位和间位的硼化产物(Scheme 6). 在邻位硼化反应 中胺和配体的选择是关键, 使用位阻较大的叔丁胺会显 著提升反应的转化率, 对此作者的解释是, 大位阻有利 于打开金属中心的空位, 实现 $\mathrm{C}-\mathrm{H}$ 键活化. 该体系下 反应具有良好的选择性，对带不同取代基的底物均未检 测到双硼化副产物. 当使用富电子的 L6 时，反应则倾 向于形成间位嗍化的产物. 虽然对于间位选择性的具体 机制并不清楚，但是作者推测可能与催化剂上的配体和 底物之间的静电相互作用, 以及 $\mathrm{B}-\mathrm{N}$ 键的二级相互作 用有关.

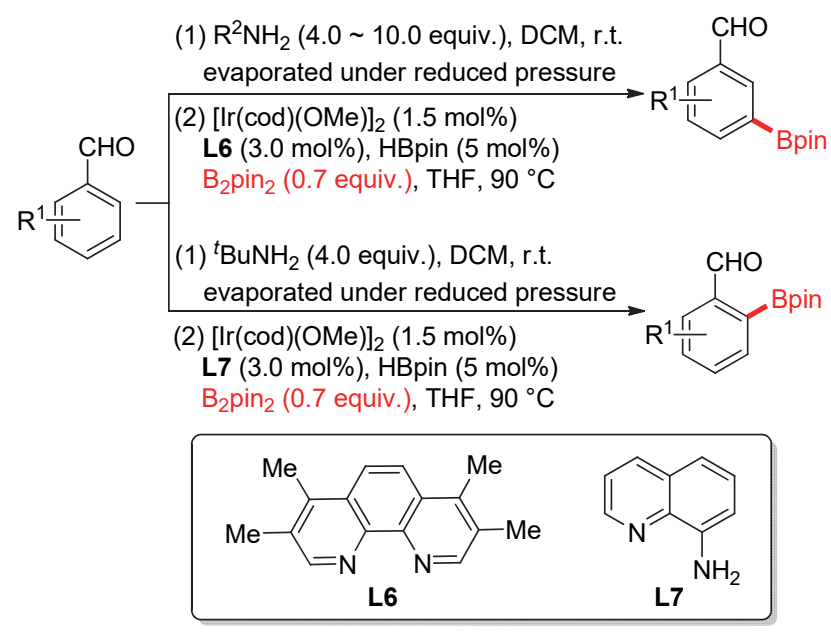

图式 6 配体调控铱催化选择性邻位/间位 $\mathrm{C}-\mathrm{H}$ 键嗍化反应 Scheme 6 Ligand-enabled iridium catalyzed selective orthoand meta- $\mathrm{C}-\mathrm{H}$ bond borylation

2014 年, Smith、Maleczka 和 Krska 等 ${ }^{[57]}$ 使用双齿 Si-P 配体 L8 与催化剂 $[\mathrm{Ir}(\mathrm{OMe})(\mathrm{cod})]_{2}$ 配位形成的富电 子活性中间体来促进 $\mathrm{C}-\mathrm{H}$ 键的断裂(Scheme 7). 他们 使用该催化体系实现了酯基导向的邻位硼化反应，对于 邻位位阻较小的底物, 检测到了相应的双硼化产物, 增

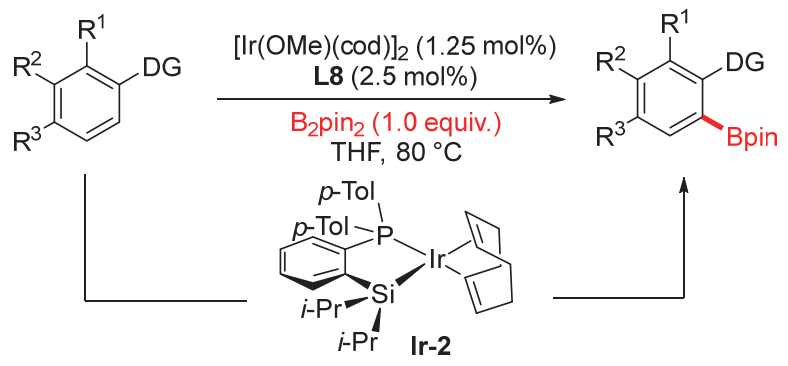

$\mathrm{DG}=\mathrm{CO}_{2} \mathrm{R}, \mathrm{CONR}$, OR, pyridine etc.

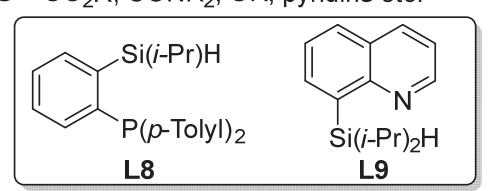

图式 7 Si-P 双齿配体参与铱催化 $\mathrm{C}-\mathrm{H}$ 键嗍化反应

Scheme 7 Iridium catalyzed $\mathrm{C}-\mathrm{H}$ bond borylation with Si-P bidentate ligand 
加导向基团的位阻可减少双嗍化的发生. 反应对于酰 胺、氨基甲酸酯、醚类以及吡啶为导向基团的底物均适 用. 当使用 Si-N 双齿配体 L9 也能以相当的收率得到叔 丁基苯甲酸酯邻位硼化的产物, 证明了硅基结构的使用 是反应发生的关键.

2017 年, 李鹏飞课题组 ${ }^{[58]}$ 设计了 N,B-双齿配体 $\mathbf{L 1 0}$, 在 $1.0 \mathrm{~mol} \%$ 的 $[\mathrm{Ir}(\mathrm{cod}) \mathrm{Cl}]_{2}$ 催化下得到了酯基导向 的邻位 $\mathrm{C}-\mathrm{H}$ 键硼化产物. 该催化体系底物范围广, 可 适用于不同导向基团, 如酚、醋酸盐、氨基甲酸酯、吡 啶、烷基胺、肜、腙等的底物(Scheme 8). 对于 2-氨基 吡啶类衍生物也能发生邻位 $\mathrm{C}\left(\mathrm{sp}^{3}\right)-\mathrm{H}$ 键硼化. 值得一 提的是，在该反应体系下，酯基作为导向基团的反应性 能要好过酰胺类化合物, 这与其他的 $\mathrm{C}-\mathrm{H}$ 键活化体系 相反. 作者分离得到了中间体 Ir-3, 证实 $\mathrm{B}-\mathrm{Si}$ 键与催 化剂发生了氧化加成.

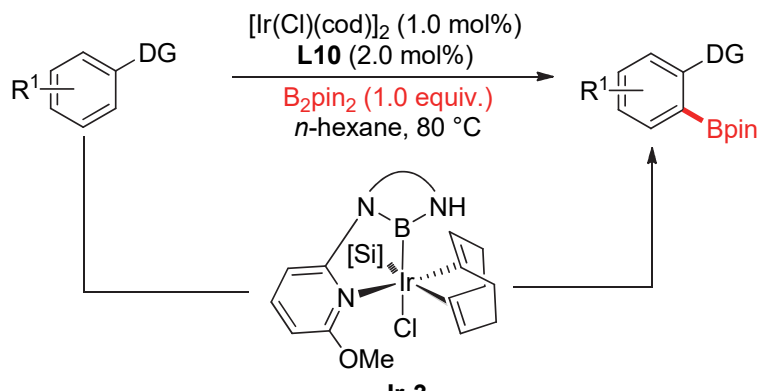

$\mathrm{DG}=\mathrm{CO}_{2} \mathrm{R}, \mathrm{OH}, \mathrm{OAc}, \mathrm{OCONR}$, pyridine, $\mathrm{CH}_{2} \mathrm{NMe}_{2}, \mathrm{CHNOR}, \mathrm{CHNR}$ etc.

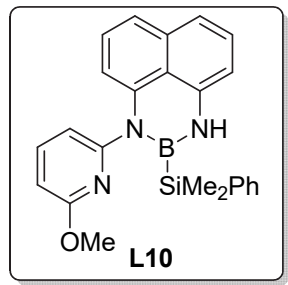

图式 8 N-B 双齿配体参与铱催化的 $\mathrm{C}-\mathrm{H}$ 键硼化反应 Scheme 8 Iridium catalyzed $\mathrm{C}-\mathrm{H}$ bond borylation with N-B bidentate ligand

具有光学活性的有机硼酸酯在药物开发和合成催 化领域 ${ }^{[59-61]}$ 有着广泛的应用, 然而通过 $\mathrm{C}-\mathrm{H}$ 键嗍化的 方法来实现不对称催化的实例却不多[62-63]. 徐森苗和柯 卓锋等[64]在 2019 年设计了手性双齿配体 L11, 并将其 成功应用到了 $\mathrm{C}\left(\mathrm{sp}^{2}\right)-\mathrm{H}$ 键的硼化反应中. 反应使用 5 $\mathrm{mol} \%$ 的 $[\mathrm{Ir}(\mathrm{Cl})(\mathrm{cod})]_{2}$ 和 $10 \mathrm{~mol} \%$ 的配体 $\mathbf{L 1 1}$ 与 $\mathrm{B}_{2} \mathrm{pin}_{2}$ 反 应，对具有潜手性的二芳基甲胺进行去对称化以及对外 消旋的底物进行了动力学拆分, 以较高的产率 $(30 \%$ 95\%)和对映选择性(79\% 96\% ee) 得到了相应的手性砋 酸酯产物(Scheme 9). 在该体系下，带吸电子官能团的 底物相较于给电子的底物会获得更高的对映选择性. 这
一体系的发展，首次实现了二芳基甲胺的不对称 $\mathrm{C}-\mathrm{H}$ 键硼化反应.

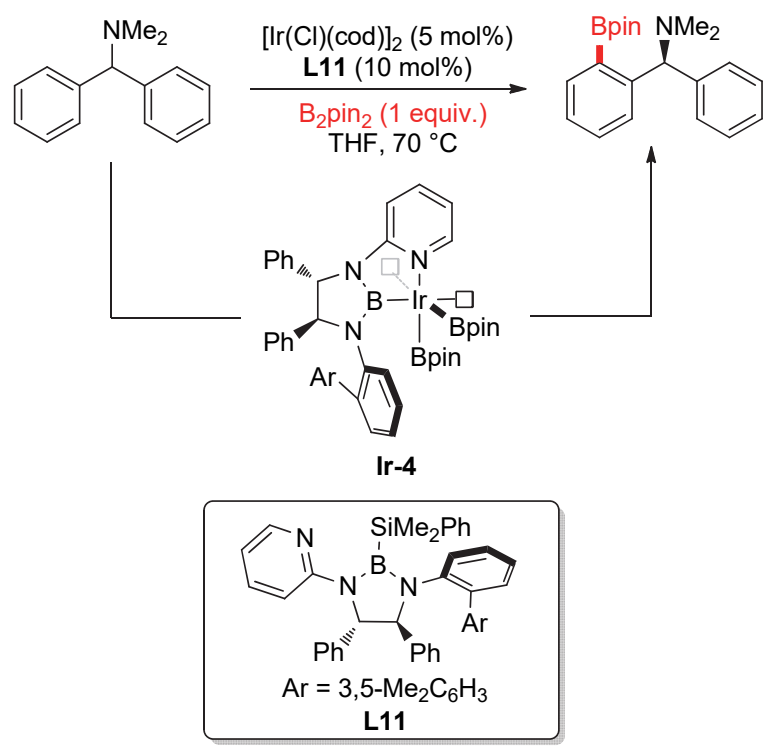

图式 9 铱催化二芳基甲胺的不对称 $\mathrm{C}-\mathrm{H}$ 键硼化反应 Scheme 9 Iridium catalyzed asymmetric $\mathrm{C}-\mathrm{H}$ borylation of diarylmethylamines

\section{3 无配体}

2019 年, 徐森苗课题组 ${ }^{[65]}$ 实现了室温下 [Ir(OMe)(cod) $]_{2}$ 催化的 2-茮基吡啶和 HBpin 的邻位 $\mathrm{C}-\mathrm{H}$ 键喼化 反应(Scheme 10). 反应对于含给电子或吸电子基团的 底物均兼容。该催化体系不需要外加配体，条件简单, 反应温和。

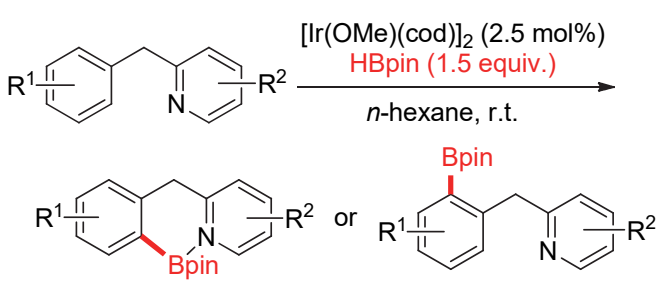

图式 10 铱催化 2-芐基吡啶的 $\mathrm{C}-\mathrm{H}$ 键喼化反应 Scheme 10 Iridium catalyzed $\mathrm{C}-\mathrm{H}$ borylation of 2-benzylpyridine

\section{2 铑催化 C一H 键硼化反应}

在元素周期表中, 金属铑与铱是同族元素，亦在导 向基团辅助的邻位官能团化反应中应用广泛，同样，在 邻位 $\mathrm{C}-\mathrm{H}$ 键硼化中也备受关注. Sawamura 等 ${ }^{[66-68]}$ 曾将 金属铱催化剂通过膦配体桥联负载到硅胶上，制备了异 相铱催化剂用于芳基酯、酰胺、磺酸酯等的邻位硣化反 应. 2011 年, 他们 ${ }^{[69]}$ 将此策略用于金属铑催化剂, 通过 二氧化硅负载的桥头单膦配体和 $[\mathrm{Rh}(\mathrm{OH})(\mathrm{COD})]_{2}$ 原位 生成膦配位的铑络合物于室温下，以 $\mathrm{B}_{2} \mathrm{pin}_{2}$ 为嗍源，快 
速催化含氮导向基团底物参与的邻位芳基 $\mathrm{C}-\mathrm{H}$ 键硣化 反应(Eq. 3). 该反应体系适用于一系列含氮导向基团， 包括饱和与不饱和的氮杂环、叔氨基烷基以及亚胺基团, 并对 $\mathrm{C}-\mathrm{H}$ 键周围空间排斥的耐受性好, 区域选择性高. 随后，他们将该反应体系扩展到 $\mathrm{C}\left(\mathrm{sp}^{3}\right)-\mathrm{H}$ 键的砋化反

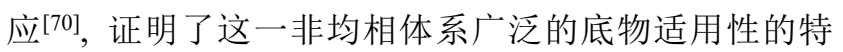
点.

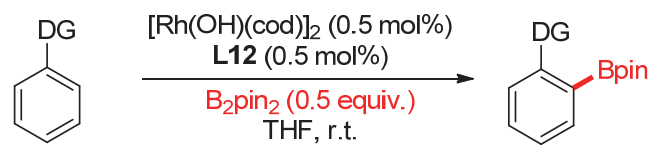

$\mathrm{DG}=$ pyridine, pyrazole, imidazole, oxazoline, $\mathrm{CH}_{2} \mathrm{NR}_{2}$ etc.

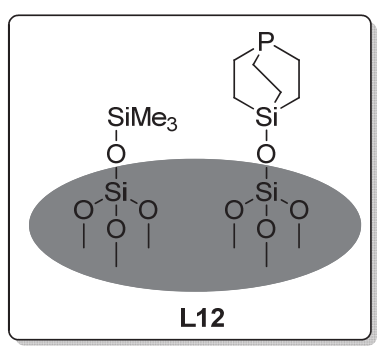

2013 年, 燕红和陈浩等 ${ }^{[71]}$ 使用 $\left[\mathrm{RhCp}^{*}(\mathrm{OTf})_{2}\right]$ 和 $\mathrm{Cu}(\mathrm{OAc})_{2}$ 双催化剂, 实现了 2-联苯基伯胺的 $\mathrm{C}-\mathrm{H}$ 键硣 化一胺化串联反应, 得到了咔唑产物(Scheme 11). 伯胺 因其小位阻与强配位能力, 一般难以直接用于导向基 团. 作者认为反应难以停留在硼化反应阶段, 正是因为 伯胺与催化剂紧密配位导致其失活, 而 $\mathrm{C}-\mathrm{B}$ 键的胺化 反应使得催化剂得以释放维持催化活性. 作者对反应体 系中各个组分的作用都进行了考察: 在没有铜盐, 只有 $\left[\mathrm{RhCp} *(\mathrm{OTf})_{2}\right]$ 存在的条件下，只检测到硼化产物，说明 $\mathrm{Rh}(\mathrm{III})$ 是催化 $\mathrm{C}-\mathrm{H}$ 键硼化的作用; 加入 $\mathrm{Cu}(\mathrm{OAc})_{2}$ 不仅 可以提高硼基产率, 而且是环化不可缺少的组分, 说明 铜盐既作为氧化剂将 $R h(I)$ 氧化成 $R h(I I I)$, 又作为 $C-N$ 键偶联反应的催化剂; 只有在加入 $\mathrm{K}_{2} \mathrm{CO}_{3}$ 的条件下才能 得到环化产物, 碱的作用是篗取 $\mathrm{N}-\mathrm{H}$ 上的质子, 促进 $\mathrm{C}-\mathrm{B}$ 键的胺化反应.

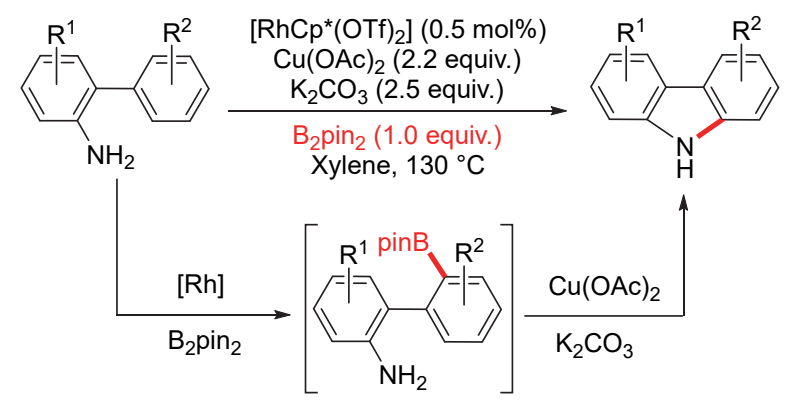

图式 11 铑催化邻氨基联芳基衍生物硼化胺化反应

Scheme 11 Rhodium catalyzed borylation-amination of 2aminobiaryl derivatives
2016 年, Miura 和 Hirano 等 ${ }^{[72]}$ 报道了 $[\mathrm{Rh}(\mathrm{OMe})-$ (cod) $]_{2}$ 催化的吡啶导向的 2-吡啶酮的 C(6)位 C-H 键硼 化反应(Eq. 4). 该催化体系无外加配体, 对不同结构的 底物的催化活性差别较大, 通过控制温度可以扩大底物 范围: 对于吡啶酮上 $\mathrm{C}(4)$ 位有甲基取代的底物，反应可 以在室温下进行; $\mathrm{C}(4)$ 位没有甲基的底物都需要 $70{ }^{\circ} \mathrm{C}$ 及以上的温度; 在吡啶酮上有苯并环时, 需要微波辅助 反应. 在参考了相关文献 ${ }^{[73-74]}$ 和机理实验的基础上，作 者虽然不能完全排除 $R h(I) / R h(I I I)$ 的机理, 但是更倾向 于 $\mathrm{Rh}(\mathrm{III}) / \mathrm{Rh}(\mathrm{V})$ 的机理循环(Scheme 12). [Rh(OMe)$(\operatorname{cod})]_{2}$ 与 $\mathrm{B}_{2} \mathrm{pin}_{2}$ 形成的 $\mathrm{Rh}(\mathrm{Bpin})_{3}$ 与芳基底物发生 $\mathrm{C}-\mathrm{H}$ 键活化, 消除一分子 HBpin, 再与另一分子的芳基底物 $\mathrm{C}-\mathrm{H}$ 键发生氧化加成得到双环 $\mathrm{Rh}(\mathrm{V})$ 中间体，最后还
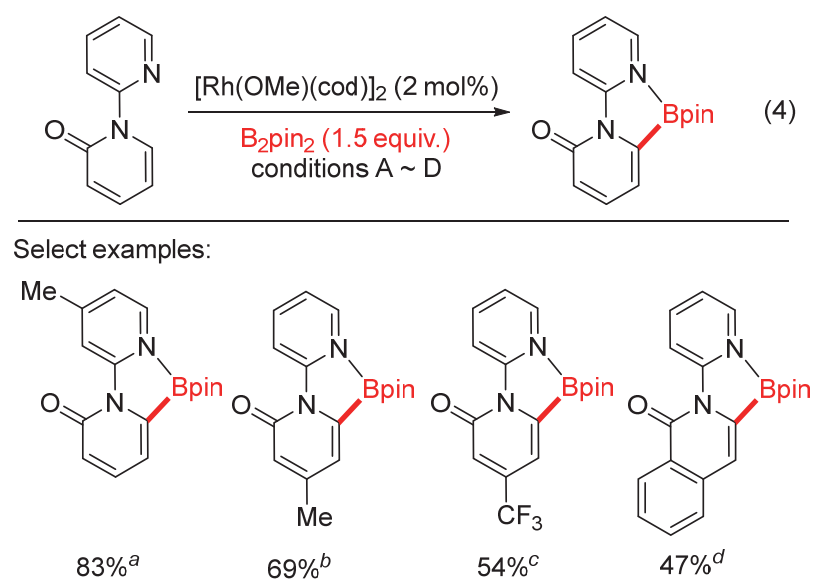

${ }^{a} \mathrm{THF}$, r.t., $24 \mathrm{~h} ;{ }^{b} \mathrm{THF}$, reflux, $4 \mathrm{~h} ;{ }^{c} \mathrm{o}$-xylene, $150{ }^{\circ} \mathrm{C}, 2 \mathrm{~h}$;

${ }^{d} \mathrm{THF}, 150^{\circ} \mathrm{C}, \mathrm{UW}, 2 \mathrm{~h}$

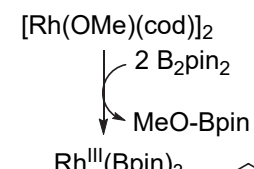

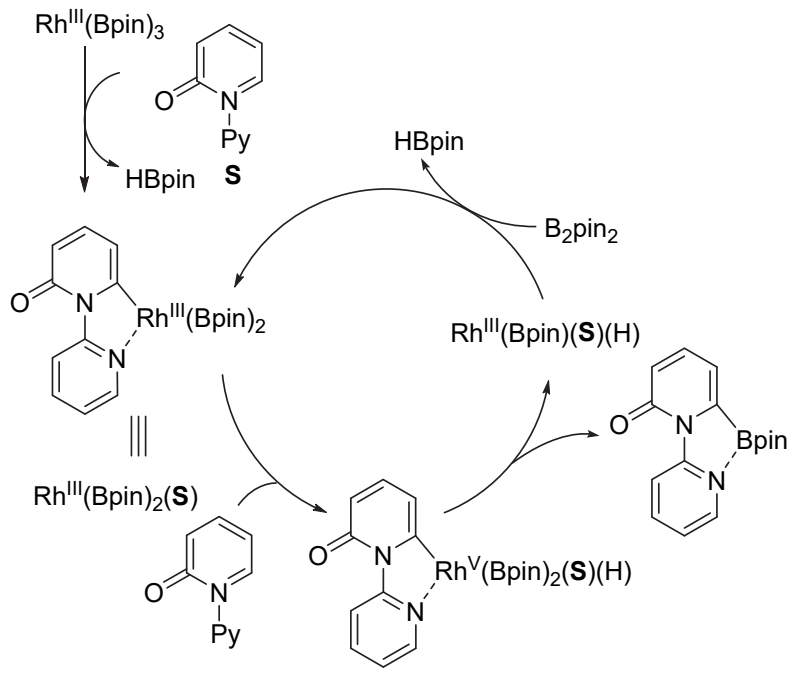

图式 12 铑催化 2-吡啶酮的嗍化反应

Scheme 12 Rhodium catalyzed borylation of 2-pyridones 
原消除后得到相应的㸴化产物, 而同时产生单环 $\mathrm{Rh}(\mathrm{III})-\mathrm{H}$ 络合物通过与 $\mathrm{B}_{2} \mathrm{pin}_{2}$ 发生配体交换完成催化 剂再生.

卡宾作为强 $\sigma$ 给电子配体, 在过渡金属催化的 $\mathrm{C}-$ $\mathrm{H}$ 键功能化方面有广泛的应用 ${ }^{[75-76]} .2014$ 年, Crudden 课 题组 ${ }^{[77]}$ 使用氮杂卡宾配位的铑催化剂 [Rh(IPr)$\left.\left(\mathrm{C}_{2} \mathrm{H}_{4}\right) \mathrm{Cl}\right]_{2}, 2$-苯基吡啶与 $\mathrm{HBpin}$ 在室温下发生脱氢嗍化 反应，选择性地得到了单硼化产物(Scheme 13, method A). 作者认为反应的活性催化剂是氮杂卡宾配位的 $\mathrm{Rh}(\mathrm{I})-\mathrm{H}$ 结构, 所以在反应体系中加入催化量的 $\mathrm{NaOEt}$ 促进催化剂前体的解聚并与 HBpin 反应脱去 ROBpin 得 到目标 $\mathrm{Rh}(\mathrm{I})-\mathrm{H}$ 催化剂. 尽管反应中所用硼试剂是过量 的, 因为反应条件温和, 产物结构中 B-N 配位阻止了吡 啶环的旋转, 所以选择性得到了单硼化产物, 产物的单 晶体结构与核磁硼谱的低位移也验证了这一点. 2019 年, 王喜存课题组 ${ }^{[78]}$ 使用 $[\mathrm{RhCl}(\mathrm{cod})]_{2}$ 和 $\mathrm{IPr}$ 卡宾配体以 及 $\mathrm{NaO}^{t} \mathrm{Bu}$ 为催化体系, 用 $\mathrm{B}_{2} \mathrm{pin}_{2}$ 为硼源进行 2-苯基吡 啶的邻位 $\mathrm{C}-\mathrm{H}$ 键单喼化反应 (Scheme 13, method B), 不同的是作者认为反应的活性催化剂是卡宾配位的三 硼基铑配合物 $\left[(\operatorname{IPr}) \operatorname{Rh}(\mathrm{BPin})_{3}\right]$.

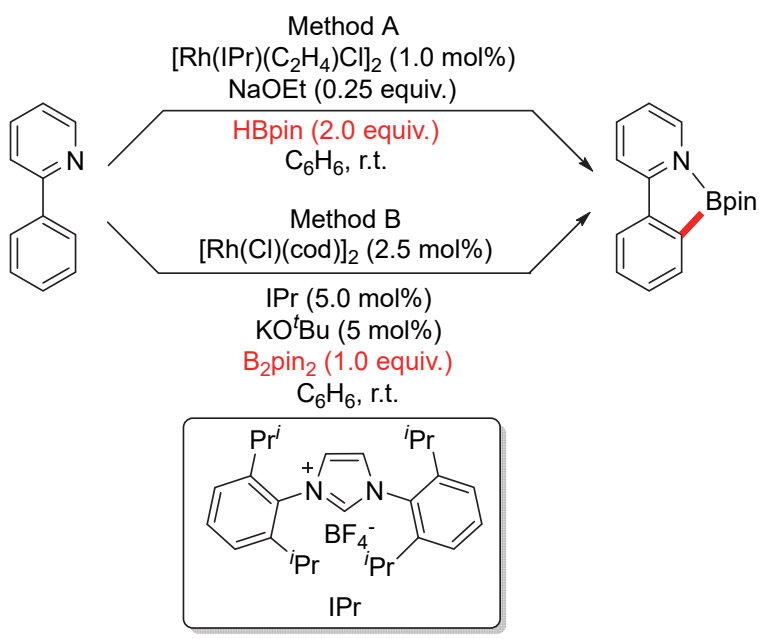

图式 13 卡宾参与铑催化 2-芳基吡啶的硼化反应

Scheme 13 Rhodium catalyzed borylation of 2-arylpyridine involved in carbene

近期, Baslé 课题组 ${ }^{[79-80]}$ 设计合成了双齿卡宾-羧酸 酯配体, 并应用到铑催化的 2-芳基吡啶的选择性邻位$\mathrm{C}-\mathrm{H}$ 键单硼化反应(Scheme 14). 卡宾与羧酸的双配位 结构可以稳定反应过程中的 Rh(III)中间体. 动力学实验 发现该反应存在一个 $45 \sim 60 \mathrm{~min}$ 的诱导期, 通过核磁监 测发现该过程产生了 $\mathrm{Rh}-\mathrm{H}$, 并伴随 $\mathrm{Cp} * \mathrm{H}$ 的生成, 据此 作者推断在诱导期通过还原消除 $\mathrm{Cp}$ * $\mathrm{H}$ 为底物的配位提 供空位. 虽然反应的机理尚未证实, 作者通过自由基捕 捉剂与采排除了自由基与异相催化的可能. 可能是因为
氮杂卡宾的大位阻效应，该催化体系对 2-芳基吡啶的取 代基的位阻效应非常敏感. 可见光作为一种绿色能源, 已被证实可以促进热反应中的决速步，例如氧化加 成 ${ }^{[81]}$ 、还原消除 ${ }^{[82]}$ 、转金属化 ${ }^{[83]}$ 等. 所以利用光反应来 代替热反应也成为 $\mathrm{C}-\mathrm{H}$ 键官能团化领域的新方向. Baslé 等 ${ }^{[84]}$ 将上述催化体系进行优化，并结合可见光诱 导，利用光能促进催化剂上的配体交换，从而使反应的 温度降低到室温. 该反应体系对于不同电性的 2-芳基吡 啶均适用，但是与缺电子基团相比，带有富电子基团的 底物产率较高. 这是首例结合可见光与金属催化实现的 导向 $\mathrm{C}-\mathrm{H}$ 键硼化反应, 相信随着对光催化机理越来越 深入的探究，会出现更多 $\mathrm{C}-\mathrm{H}$ 键官能团化的策略.
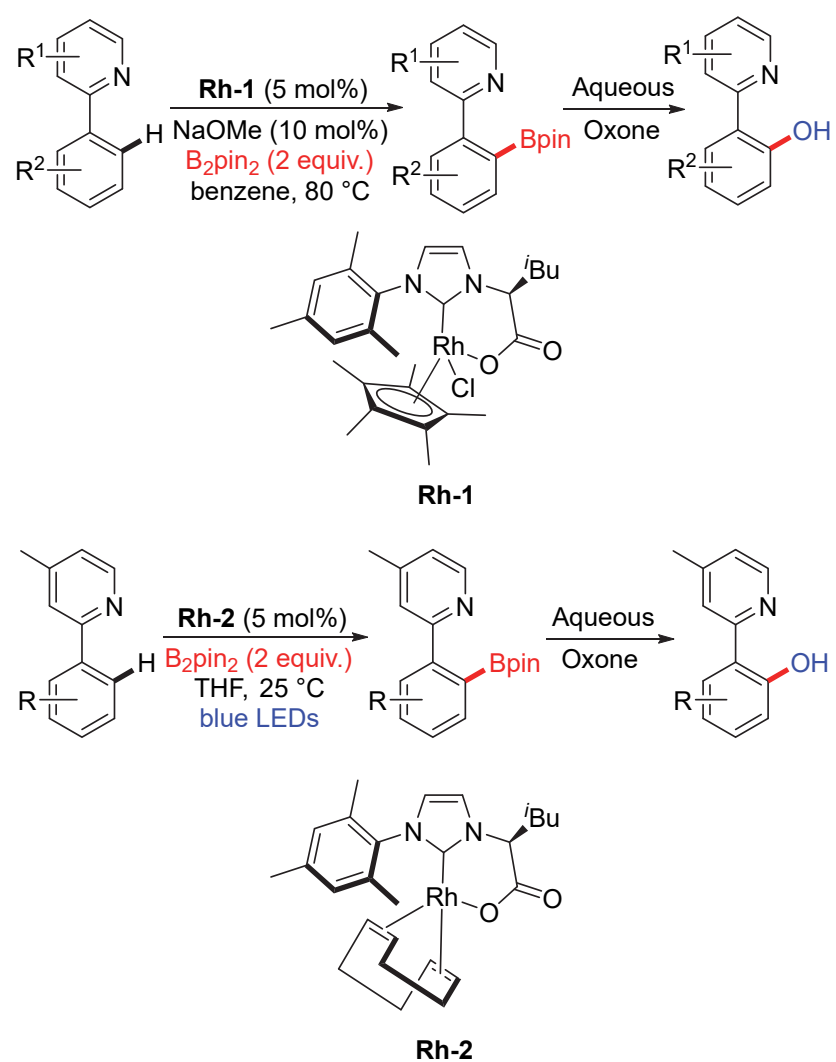

图式 14 卡宾-羧酸酯配位的铑催化 2-芳基吡啶的硼化反应 Scheme 14 Carboxyalkyl-NHC-rhodium catalyzed borylation of 2-arylpyridine

\section{3 钯催化 C-H 键硼化反应}

钯催化剂在许多反应中以其优异的反应活性、可控 的选择性、高效率和官能团耐受性而备受关注 ${ }^{[85-87]}$, 但 是因为芳基硼酸酯容易与 $\operatorname{Pd}(\mathrm{II})$ 发生转金属化 ${ }^{[88-91]}$, 所 以钯催化芳香化合物的 $\mathrm{C}-\mathrm{H}$ 键硼化反应相对较少.

2012 年，余金权等 ${ }^{[92]}$ 通过氧化 $\mathrm{C}-\mathrm{H}$ 键活化的形式 实现了 $\mathrm{Pd}(\mathrm{OAc})_{2}$ 催化 $N$-芳基苯甲酰胺衍生物与 $\mathrm{B}_{2} \mathrm{pin}_{2}$ 
的邻位 $\mathrm{C}-\mathrm{H}$ 键硼化反应(Eq. 5). 反应中弱碱 $\mathrm{TsONa}$ 的 加入是为了减少嗍试剂和硼化产物的分解. 作者推测该 反应的机理是(Scheme 15): Pd(II) 首先与芳香底物发生 $\mathrm{C}-\mathrm{H}$ 键活化产生环钯中间体 A. 第一条路径是该中间 体 $\mathbf{A}$ 先与 $\mathrm{B}_{2} \mathrm{pin}_{2}$ 经过转金属化得到的 Pd(II)-B 物种, 被 $\mathrm{K}_{2} \mathrm{~S}_{2} \mathrm{O}_{8}$ 氧化成 $\mathrm{Pd}(\mathrm{IV})-\mathrm{B}$ 物种后, 再还原消除得到最终 的产物并再生 $\operatorname{Pd}(\mathrm{II})$; 第二条路径是 $\mathrm{Pd}(\mathrm{II})-\mathrm{B}$ 物种先还 原消除得到硼化产物和 $\operatorname{Pd}(0)$, 再被氧化成 $\operatorname{Pd}(\mathrm{II})$ 进入下 一个循环; 第三条路径是中间体 $\mathbf{A}$ 先被氧化成 $\operatorname{Pd}(\mathrm{IV})$, 再经转金属化还原消除得到 $\mathrm{Pd}(\mathrm{II})$. 由于反应中并未检 测到中间体 $\mathbf{D}$ 还原消除后的产物, 所以作者更倾向于路 径 I 和路径 II.
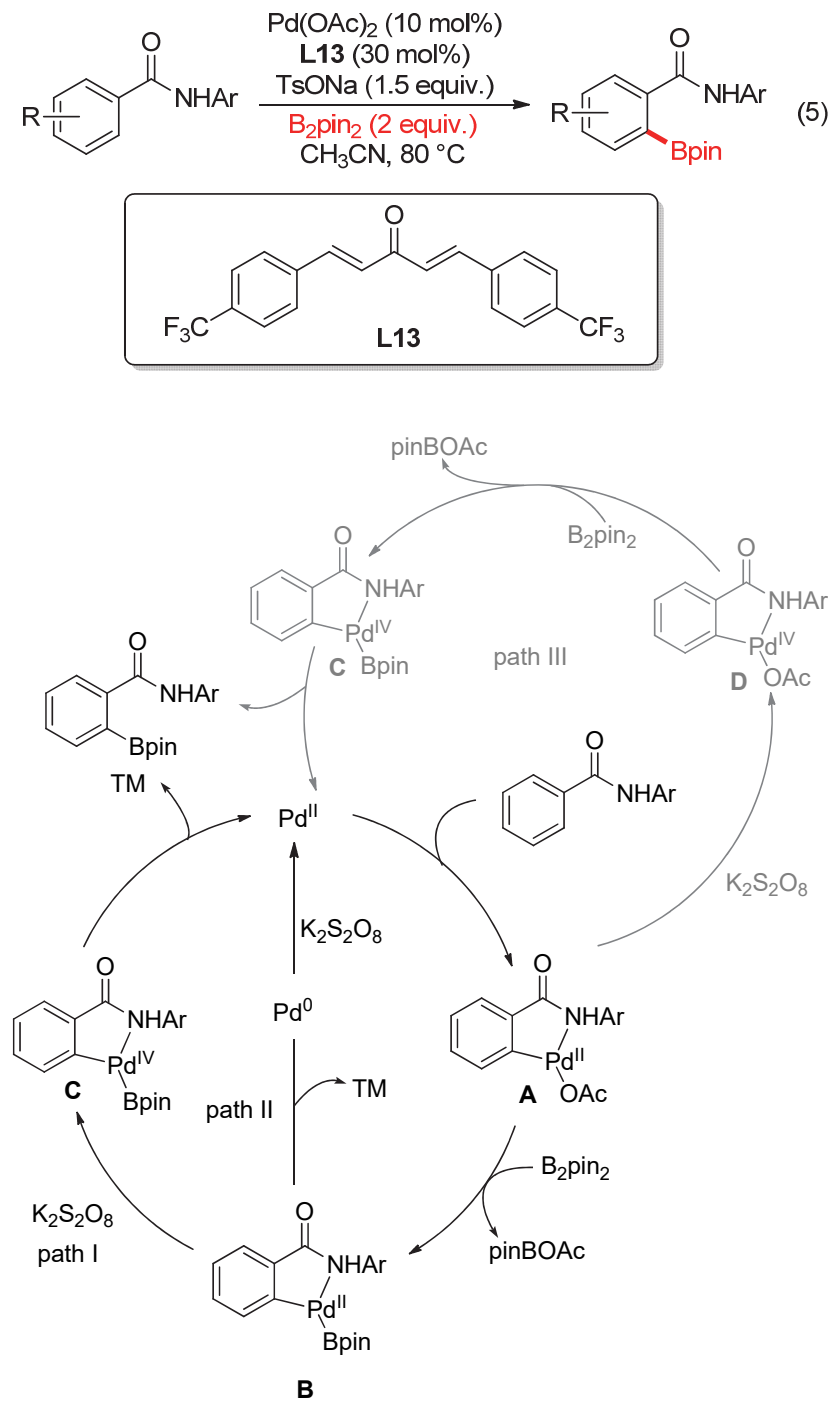

图式 15 钯催化氧化 $\mathrm{C}-\mathrm{H}$ 键硼化反应的机理

Scheme 15 Mechanism of palladium catalyzed oxidative $\mathrm{C}-\mathrm{H}$ bond borylation

2013 年, Kuninobu 和 Kanai 等 ${ }^{[93]}$ 通过底物分子间形 成 Lewis 酸碱对的策略, 实现了室温下钯催化的 2-苯基 吡啶的邻位硼化反应(Scheme 16, a). 通过 ${ }^{11}$ B NMR 证
明了 Lewis 酸性较强的 9-硼双环[3.3.1]壬烷(9-BBN)可 以与 2-苯基吡啶于室温下通过 B-N 相互作用形成络合 物. 作者认为络合物中的 $\mathrm{B}-\mathrm{H}$ 键是可以与 $\mathrm{Pd}(0)$ 发生氧 化加成生成硼基 $\mathrm{Pd}-\mathrm{H}$ 物种, 然后经过邻位 $\mathrm{C}-\mathrm{H}$ 键活化 释放一分子 $\mathrm{H}_{2}$, 最后还原消除完成催化循环. 此外, 作 者还发现，在无催化剂的情况下将 B-N 络合物加热至 $135{ }^{\circ} \mathrm{C}$, 也可以以不错的收率得到 2-苯基吡啶邻位硼化 的产物(Scheme 16, b). 据此作者推断无催化剂反应是 自由基机理, B-N 络合物在加热条件下发生 B- $\mathrm{H}$ 键均 裂产生嗍自由基与氢原子自由基, 硼原子与苯环发生亲 电取代得到相应产物. 2017 年, Kuninobu 等 ${ }^{[94]}$ 将该反应 进行了进一步优化，加入 $5 \mathrm{~mol} \%$ 的 $\mathrm{FeBr}_{3}$ 可以将反应温 度由 $135{ }^{\circ} \mathrm{C}$ 降低到 $90{ }^{\circ} \mathrm{C}$. 在紫外光下不同物态的反应 产物呈现不同苂光，展现了其在光电材料方面的潜在应 用.

(a) Low temperature (in the presence of a palladium catalyst)

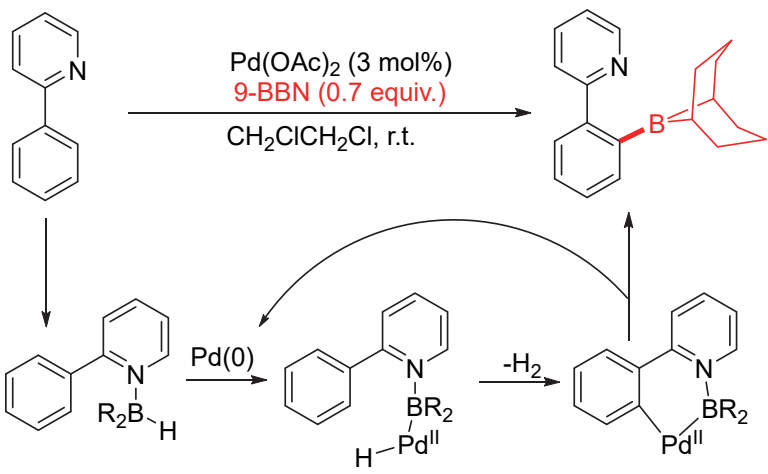

(b) High temperature (without catalyst)

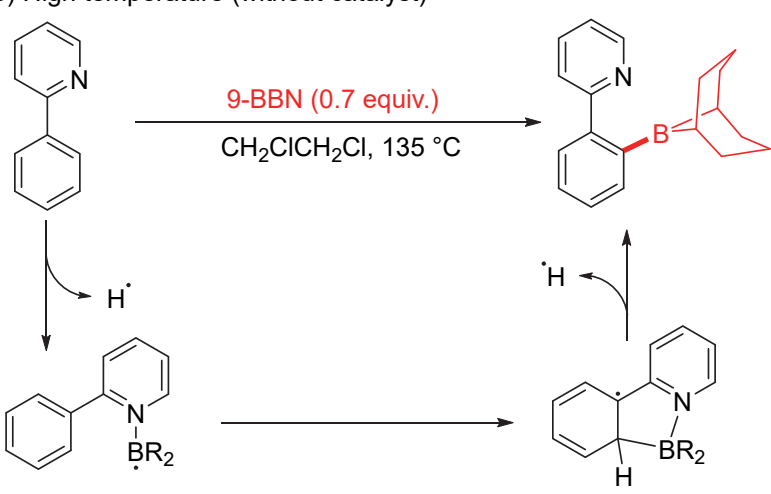

图式 16 钯催化/无催化的 2-苯基吡啶的邻位 $\mathrm{C}-\mathrm{H}$ 嗍化反应 的机理

Scheme 16 Mechanisms of palladium/free catalyzed $\mathrm{C}-\mathrm{H}$ bond borylation of 2-phenyl pyridine

\section{4 钉催化 C-H 键嗍化反应}

相比铱、铑、钯等其他贵金属，过渡金属钉相对廉 价，且在各种导向基团辅助的 $\mathrm{C}-\mathrm{H}$ 键功能化反应中表 现良好而备受关注 ${ }^{[95-97]}$, 然而钉催化的邻位芳基 $\mathrm{C}-\mathrm{H}$ 键的嗍化反应研究却相对较少. 
2014 年, Nolan 课题组 ${ }^{[98]}$ 通过 $\left[\mathrm{RuCl}\left(\mathrm{PPh}_{3}\right)_{2}\left(\eta^{5}-3-\right.\right.$ phenylindenyl)]和硅烷 $\mathrm{R}_{3} \mathrm{SiH}$ 的反应得到一系列含有硅 基 $\mathrm{Ru}-\mathrm{H}$ 结构的配合物 $\left[\mathrm{RuH}_{2}\left(\mathrm{PPh}_{3}\right)\left(\eta^{5}\right.\right.$-3-phenyl-indenyl)$\left(\mathrm{SiR}_{3}\right)$ ], 并将其用于催化 2-芳基吡啶的邻位硼化反应 (Eq. 6). 其中 Ru-1 的催化活性最高, 以 $1.5 \mathrm{~mol} \%$ 的该催 化剂, $\mathrm{B}_{2} \mathrm{pin}_{2}$ 为硼源, 可以得到 2-芳基吡啶的邻位硼化 产物. 虽然未能分离得到钉催化剂与 2-苯基吡啶发生 $\mathrm{C}-\mathrm{H}$ 键活化的中间体, 但是作者通过核磁与 GC-MS 观 测到 $\mathrm{Et}_{3} \mathrm{SiH}$, 以及在加入 $\mathrm{B}_{2} \mathrm{pin}_{2}$ 后产生的 HBpin. 据此 作者推测反应机理(Scheme 17): 催化剂与底物发生 C$\mathrm{H}$ 键活化, 并释放 $\mathrm{Et}_{3} \mathrm{SiH}$; 再与 $\mathrm{B}_{2} \mathrm{pin}_{2}$ 发生转金属化, 并释放 HBpin; 最后, 还原消除得到产物与活性催化剂, 完成催化循环.
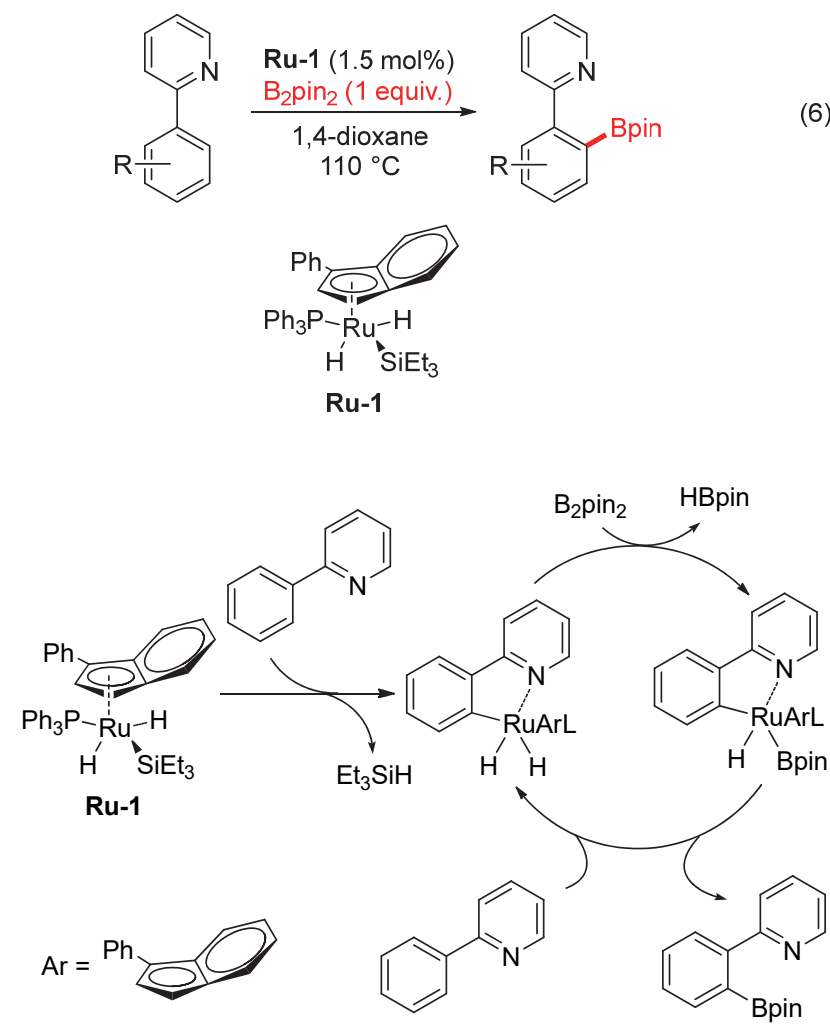

图式 $17\left[\mathrm{RuH}_{2}\left(\mathrm{PPh}_{3}\right)\left(\eta^{5}\right.\right.$-3-phenylindenyl)(SiEt 3$\left.)\right]$ 催化的 2-苯 基吡啶的 $\mathrm{C}-\mathrm{H}$ 键嗍化反应的机理

Scheme 17 Mechanisms of $\mathrm{C}-\mathrm{H}$ bond borylation of 2-phenyl pyridine catalyzed by $\left[\mathrm{RuH}_{2}\left(\mathrm{PPh}_{3}\right)\left(\eta^{5}-3\right.\right.$-phenylindenyl $\left.)\left(\mathrm{SiEt}_{3}\right)\right]$

2015 年, Murata 课题组 ${ }^{[99]}$ 使用商业可得的 $\left[\mathrm{RuH}_{2}-\right.$ $(\mathrm{CO})\left(\mathrm{PPh}_{3}\right)_{3}$ ] 为催化剂, 实现了 2-芳基吡啶与 HBpin 的 邻位 $\mathrm{C}-\mathrm{H}$ 键双硼化产物, 反应中检测到微量的单喼化 产物(Eq. 7). 该反应无需氢受体, 还可以使用 $\mathrm{B}_{2} \mathrm{pin}_{2}$ 作 为硼源. 只有当 2-芳基吡啶的苯环其中一个邻位 $\mathrm{C}-\mathrm{H}$ 键被取代, 或者单嗍化产物因为位阻不能自由旋转时, 才能获得单硼化产物. 作者通过 2-苯基吡啶的同位素标 记与动力学实验发现, $\mathrm{C}-\mathrm{H}$ 键活化步骤是个平衡过程,
并不是决速步. 结合密度泛函理论(DFT)计算作者推测 反应的机理是 (Scheme 18): 首先, 由催化剂前驱体 $\left[\mathrm{RuH}_{2}(\mathrm{CO})\left(\mathrm{PH}_{3}\right)_{3}\right]$ 产生活性催化剂 $\mathrm{Ru}(0)$; 然后 2-芳基吡 啶与 $\mathrm{Ru}(0)$ 配位, 并通过氧化加成完成邻位 $\mathrm{C}-\mathrm{H}$ 键的 活化，生成芳基 $\mathrm{Ru}(\mathrm{II})-\mathrm{H}$ 物种; 接着, HBpin 通过配体交 换与芳基 $\mathrm{Ru}(\mathrm{II})-\mathrm{H}$ 物种配位，并通过 $\sigma$-键复分解反应脱 出一分子 $\mathrm{H}_{2}$ 生成芳基 $\mathrm{Ru}(\mathrm{II})-\mathrm{Bpin}$ 络合物; 最后，还原消 除得到目标产物并再生活性催化剂 $\mathrm{Ru}(0)$, 完成催化循 环. 其中, $\sigma$-键复分解反应活化能最高, 为反应的决速 步.
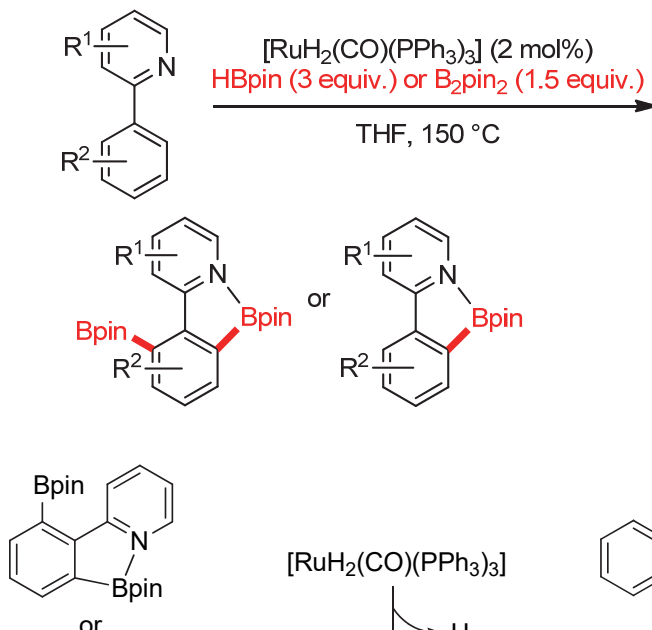<smiles></smiles>

$\left[\mathrm{RuH}_{2}(\mathrm{CO})\left(\mathrm{PPh}_{3}\right)_{3}\right]$<smiles></smiles><smiles>[3H][13CH3]</smiles><smiles>[R]O[Pb]</smiles>

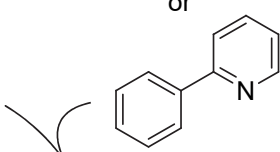

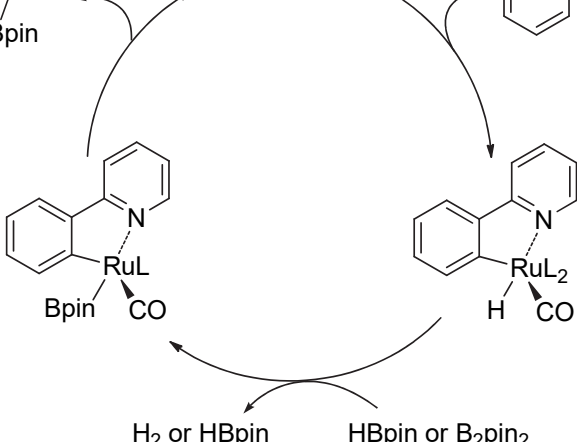

图式 $18\left[\mathrm{RuH}_{2}(\mathrm{CO})\left(\mathrm{PPh}_{3}\right)_{3}\right]$ 催化的 $\mathrm{C}-\mathrm{H}$ 键双硼化反应的机 理

Scheme 18 Mechanism of $\mathrm{C}-\mathrm{H}$ bond diborylation catalyzed by $\left[\mathrm{RuH}_{2}(\mathrm{CO})\left(\mathrm{PPh}_{3}\right)_{3}\right]$

2017 年, Ackermann 课题组 ${ }^{[100]}$ 以空气稳定的 $[\mathrm{Ru}-$ $\left(\mathrm{O}_{2} \mathrm{CMes}\right)\left(p\right.$-cymene)]为催化剂前体, $\mathrm{B}_{2} \mathrm{pin}_{2}$ 为嶰试剂, 实现了吡啶导向的邻位 $\mathrm{C}-\mathrm{H}$ 键硼化反应(Eq. 8). 值得 一提的是，该反应体系对于 $\mathrm{C}\left(\mathrm{sp}^{3}\right)-\mathrm{H}$ 也同样适用，例 如吡咯烷、哌啶、环己亚胺以及直链的烷基胺等. 作者 进行了一系列的机理研究实验: 动力学实验发现反应存 在一个诱导期, 说明 $\left[\mathrm{Ru}\left(\mathrm{O}_{2} \mathrm{CMes}\right)(p\right.$-cymene $\left.)\right]$ 需要活化 生成活性催化剂; 自由基捕捉剂以及金属采都不能完全 
阻断反应进行, 说明该反应既不是自由基机理也不属于 异相催化的范畴; 化学计量反应的核磁检测发现催化剂 前体可以与 HBpin 作用, 进而对底物砋化, 作者猜测可 能是羧酸修饰的嗍基金属钬团簇才是活性催化剂. 此 外，作者合成并证明了芳基环钉物种具有很好的催化活 性.

$$
\text { (Het) } \mathrm{Ar}-\mathrm{H} \underset{\left[\mathrm{Ru}\left(\mathrm{O}_{2} \mathrm{CMes}\right)(\text { p-cymene })\right](5.0 \mathrm{~mol} \%)}{\stackrel{\mathrm{B}_{2} \mathrm{pin}_{2}(1.5 \text { equiv. })}{1,4 \text {-dioxane, } 110^{\circ} \mathrm{C}}}(\mathrm{Het}) \mathrm{Ar}-\mathrm{Bpin} \text { (8) }
$$

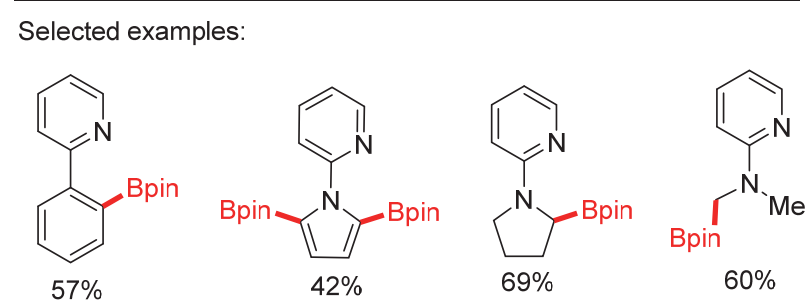

2018 年, Murata 课题组 ${ }^{[101]}$ 使用零价钉 $\mathrm{Ru}(\operatorname{cod})(\cot )$ 为催化剂前体, 实现了芳基亚胺的邻位 $\mathrm{C}-\mathrm{H}$ 硼化反应, 并通过使用不同的嗍源以及调节其用量选择性, 得到单 硼化或双嗍化反应产物(Scheme 19).
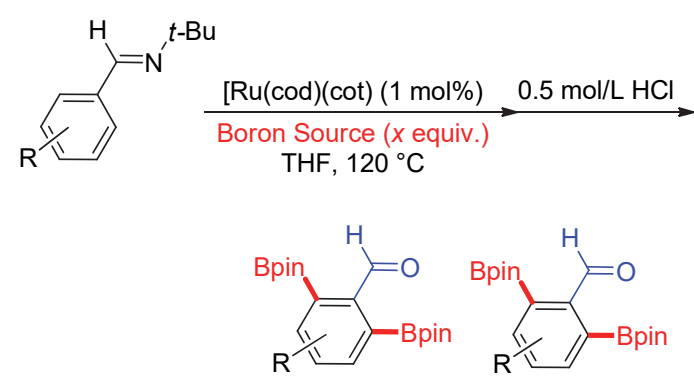

$$
\begin{array}{ll}
\text { HBpin (2 equiv.) } & 82 \% \\
\mathrm{~B}_{2} \operatorname{pin}_{2}(0.76 \text { equiv.) } & 48 \% \\
\mathrm{~B}_{2} \operatorname{pin}_{2}(1.52 \text { equiv.) } & 4 \%
\end{array}
$$

$$
\begin{gathered}
0 \% \\
5 \% \\
64 \%
\end{gathered}
$$

图式 $19 \mathrm{Ru}(\operatorname{cod})(\cot )$ 催化芳基亚胺的 $\mathrm{C}-\mathrm{H}$ 键嗍化反应 Scheme $19 \mathrm{C}-\mathrm{H}$ bond borylation of aromatic imines catalyzed by $\mathrm{Ru}(\operatorname{cod})(\cot )$

最近, 姚武冰等 ${ }^{[102]}$ 设计并合成了一种 PCP 钳式配 体配位的高活性钌催化剂 $\left({ }^{i} \mathrm{PrPOCOP}\right) \mathrm{Ru}$, 以 $0.5 \mathrm{~mol} \%$ 的催化剂用量, 通过控制 $\mathrm{B}_{2} \mathrm{pin}_{2}$ 用量选择性地得到双硼 化或者单硼化产物(Eq. 9). 作者猜想该体系可以选择性 得到双硼化的产物, 是因为所设计的催化剂具有高的热 稳定性和活性, 在 $150{ }^{\circ} \mathrm{C}$ 反应温度下可以克服 $\mathrm{B}-\mathrm{N}$ 间 的相互作用, 促使芳环上的另一个邻位 $\mathrm{C}-\mathrm{H}$ 键发生嗍 化反应. 通过对比实验以及动力学试验, 作者排除了自 由基反应的机理以及异相催化, 推理该反应机理是 (Scheme 20): 首先, $\left({ }^{i} \mathrm{PrPOCOP}\right) \mathrm{Ru}$ 脱去 1,4-环庚二烯配 体后形成配位不饱和的 $\mathrm{Ru}-\mathrm{H}$ 物种; 然后, 该 $\mathrm{Ru}-\mathrm{H}$ 与
$\mathrm{B}_{2} \mathrm{pin}_{2}$ 发生配位，与 $\sigma$-键复分解反应形成关键 $\mathrm{Ru}-\mathrm{B}$ 中间 体; 接着, $\mathrm{Ru}-\mathrm{B}$ 中间体与底物 2-芳基吡啶发生第二次 $\sigma$ 键复分解反应得到目标产物, 并再生催化剂. 除了 2-芳 基吡啶类的底物, 2-酚基吡啶类底物也适用.
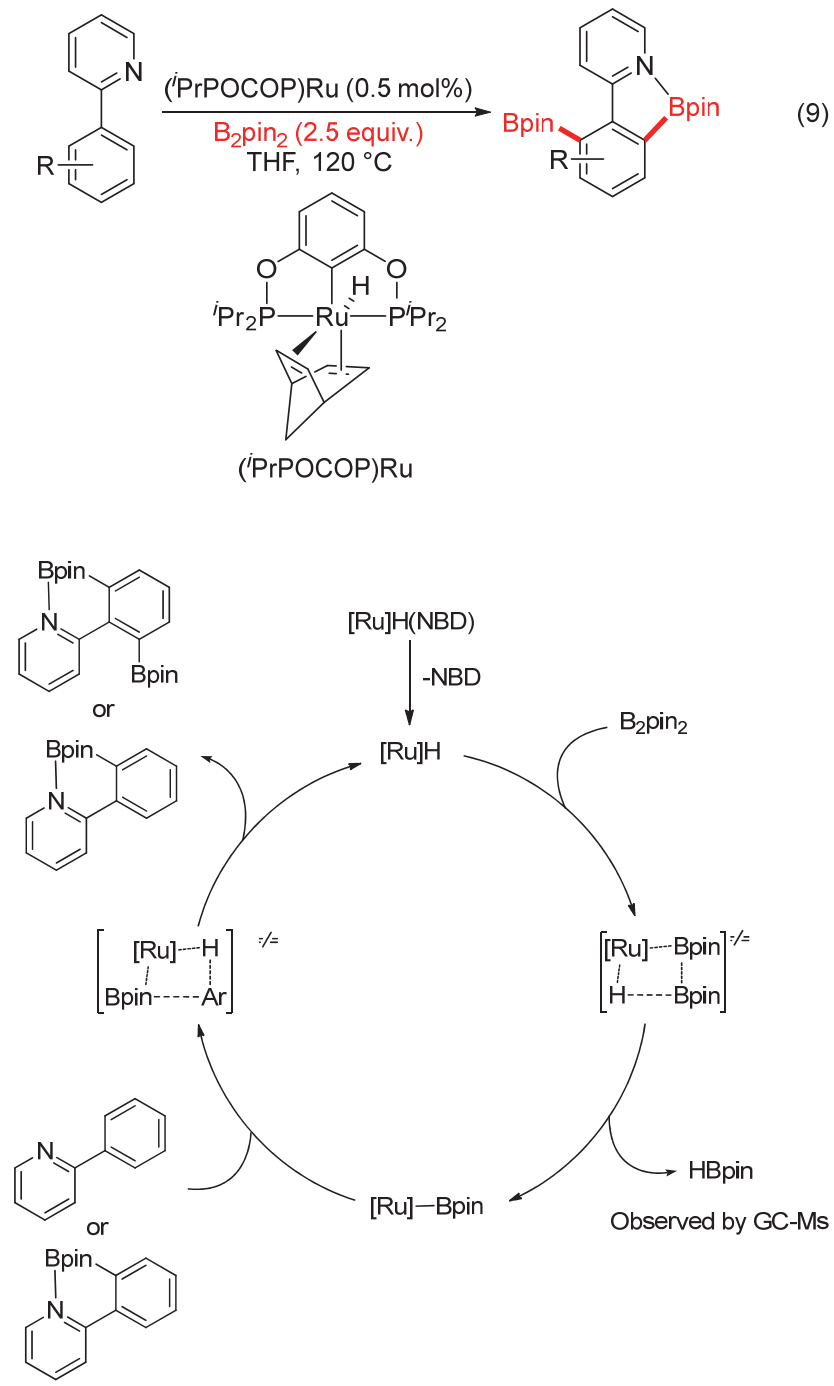

图式 20 ( $\left.{ }^{i} \mathrm{PrPOCOP}\right) \mathrm{Ru}$ 催化的 $\mathrm{C}-\mathrm{H}$ 键双嗍化反应的机理 Scheme 20 Mechanism of $\mathrm{C}-\mathrm{H}$ bond diborylation catalyzed by $\left({ }^{i} \mathrm{PrPOCOP}\right) \mathrm{Ru}$

\section{5 结论与展望}

综述了过渡金属催化的氮原子导向的芳香化合物 的 $\mathrm{C}\left(\mathrm{sp}^{2}\right)-\mathrm{H}$ 键硼化反应. 该催化反应逐渐向更加温和 的条件、更高的选择性和更廉价的金属催化剂方向发展. 但目前 $\mathrm{C}-\mathrm{H}$ 键硼化仍然存在一些限制: (1)多数反应体 系得到单双取代混合物，反应的选择性有待进一步提 高; (2)大多数催化体系对高极性官能团的兼容性比较 差; (3)通过 $\mathrm{C}-\mathrm{H}$ 键转化直接获得手性硼化产物的反应 相对较少. 通过对反应机理更加深入的研究, 发展新的 催化概念和策略，例如结合可见光催化、电催化等更加 
绿色优质的催化方法，是该领域今后的发展方向.

\section{References}

[1] Darses, S.; Genet, J.-P. Chem. Rev. 2008, 108, 288

[2] Fyfe, J. W. B.; Watson, A. J. B. Chem 2017, 3, 31.

[3] Shi, D.-F.; Wang, L.; Xia, C.-G.; Liu, C. Chin. J. Org. Chem. 2020, 40,3605 (in Chinese) (史敦发, 王露, 夏春谷, 刘超, 有机化学, 2020, 40, 3605.)

[4] Wang, L.-J.; Sheng, X.-L.; Wang, J.; Zhang, Y.-H. Chin. J. Org Chem. 2021, 41, 567 (in Chinese). (王李娟, 盛显良, 王杰, 张玉辉, 有机化学, 2021, 41, 567.)

[5] Kiprof, P.; Carlson, J. C.; Anderson, D. R.; Nemykin, V. N. Dalton Trans. 2013, 42, 15120

[6] Zou, L. Y.; Zhang, Z. L.; Ren, A. M.; Ran, X. Q.; Feng, J. K. Theor. Chem. Acc. 2010, 126, 361.

[7] Hunt, C. D. J. Trace Elem. Med. Biol. 2012, 26, 157.

[8] Morgan, J.; Pinhey, J. T. J. Am. Chem. Soc. 1990, 3, 715.

[9] Wulff, G.; Lauer, M. J. Organomet. Chem. 1983, 256, 1.

[10] Ishiyama, T.; Murata, M.; Miyaura, N. J. Org. Chem. 1995, 60, 7508 .

[11] Kubota, K.; Iwamoto, H.; Ito, H. Org. Biomol. Chem. 2017, 15, 285.

[12] Xu, Y.-L.; Fang, H. Chin. J. Org. Chem. 2018, 38, 738 (in Chinese). (徐玉良, 方浩, 有机化学, 2018, 38, 738.)

[13] Iqbal, S. A.; Pahl, J.; Yuan, K.; Ingleson, M. J. Chem. Soc. Rev. 2020, 49, 4564

[14] Li, Y.; Wu, X. F. Angew. Chem., Int. Ed. 2020, 59, 1770.

[15] Hartwig, J. F. Chem. Soc. Rev. 2011, 40, 1992.

[16] Ros, A.; Fernández, R.; Lassaletta, J. M. Chem. Soc. Rev. 2014, 43, 3229.

[17] Liao, G.; Wu, Y.-J.; Shi, B.-F. Acta Chim. Sinica 2020, 78, 289 (in Chinese). (廖港, 吴勇杰, 史炳锋, 化学学报, 2020, 78, 289.)

[18] Zou, X.-L.; Xu, S.-M. Chin. J. Org. Chem. 2021, 41, 2610 (in Chinese) (邹晓亮, 徐森苗, 有机化学, 2021, 41, 2610.)

[19] Liu, L.-H.; Du, R.-R.; Xu. S.-M. Chin. J. Org. Chem. 2021, 41, 1572 (in Chinese) (刘路华, 杜荣荣, 徐森苗, 有机化学, 2021, 41, 1572.)

[20] Nguyen, P.; Blom, H. P.; Westcott, S. A.; Taylor, N. J.; Marder, T. B. J. Am. Chem. Soc. 1993, 115, 9329.

[21] Waltz, K. M.; He, X.; Muhoro, C.; Hartwig, J. F. J. Am. Chem. Soc. 1995, $117,11357$.

[22] Mkhalid, I. A. I.; Barnard, J. H.; Marder, T. B.; Murphy, J. M.; Hartwig, J. F. Chem. Rev. 2010, 110, 890.

[23] Wu, M.; Huang, X.-P.; Zhang, H.-B.; Li, P.-F. Chin. J. Org. Chem. 2019, 39, 3114 (in Chinese). (吴梅, 黄新平, 张海兵, 李鹏飞, 有机化学, 2019, 39, 3114.)

[24] Jiang, X.-L.; Hao, J.-Q.; Zhou, G.-Q.; Hou, C.-C.; Hu, F.-D. Chin. J. Org. Chem. 2019, 39, 1811 (in Chinese). (姜晓蕾, 郝佳奇, 周国庆, 侯程程, 胡芳东, 有机化学, 2019, 39, 1811.)

[25] Wu, Y.-J.; Shi, B.-F. Chin. J. Org. Chem. 2020, 40, 3517 (in Chinese). (吴勇杰，史炳锋，有机化学, 2020, 40, 3517.)

[26] Luo, F.-H. Chin. J. Org. Chem. 2019, 39, 3084 (in Chinese). (罗飞华, 有机化学, 2019, 39, 3084.)

[27] Cheng, H.-C.; Lin, J.-L.; Zhang, Y.-F.; Chen, B.; Wang, M.; Cheng, L.-H.; Ma, J.-L. Chin. J. Org. Chem. 2019, 39, 318 (in Chinese). (程辉成, 林锦龙, 张耀丰, 陈冰, 王敏, 程丽华, 马姣丽, 有机 化学, 2019, 39, 318.)

[28] Liu, Y.-H.; Xia, Y.-N.; Shi, B.-F. Chin. J. Chem. 2020, 38, 635.

[29] Boebel, T. A.; Hartwig, J. F. J. Am. Chem. Soc. 2008, 130, 7534.

[30] Wright, S. E.; Richardson-Solorzano, S.; Stewart, T. N.; Miller, C. D.; Morris, K. C.; Daley, C. J. A.; Clark, T. B. Angew. Chem., Int. Ed. 2019, 58, 2834

[31] Wen, J.; Wang, D.; Qian, J.; Wang, D.; Zhu, C.; Zhao, Y.; Shi, Z.
Angew. Chem., Int. Ed. 2019, 58, 2078.

[32] Fukuda, K.; Iwasawa, N.; Takaya, J. Angew. Chem., Int. Ed. 2019, $58,2850$.

[33] Xu, F.; Duke, O. M.; Rojas, D.; Eichelberger, H. M.; Kim, R. S.; Clark, T. B.; Watson, D. A. J. Am. Chem. Soc. 2020, 142, 11988

[34] Su, B.; Hartwig, J. F. Angew. Chem., Int. Ed. 2018, 57, 10163.

[35] Sumida, Y.; Harada, R.; Sumida, T.; Hashizume, D.; Hosoya, T. Chem. Lett. 2018, 47, 1251.

[36] Chattopadhyay, B.; Dannatt, J. E.; Andujar-De Sanctis, I. L.; Gore, K. A.; Maleczka, R. E.; Singleton, D. A.; Smith, M. R. J. Am. Chem. Soc. 2017, 139, 7864.

[37] Mihai, M. T.; Williams, B. D.; Phipps, R. J. J. Am. Chem. Soc. 2019, 141,15477.

[38] Li, D.; Zhang, H.; Wang, Y. Chem. Soc. Rev. 2013, 42, 8416.

[39] Rao, Y.; Amarne, H.; Wang, S. Chem. Rev. 2012, 256, 759.

[40] Haque, A.; Al-Balushi, R. A.; Raithby, P. R.; Khan, M. S. Molecules 2020, 25, 2645.

[41] Dou, C.; Liu, J.; Wang, L. Sci. China Chem. 2017, 60, 450.

[42] Zhao, C.; Wang, J.; Jiao, J.; Huang, L.; Tang, J. J. Mater. Chem. C 2020, $8,28$.

[43] Iverson, C. N.; Smith, M. R. J. Am. Chem. Soc. 1999, 121, 7696.

[44] Ishiyama, T.; Takagi, J.; Ishida, K.; Miyaura, N.; Anastasi, N. R.; Hartwig, J. F. J. Am. Chem. Soc. 2002, 124, 390.

[45] Ishiyama, T.; Takagi, J.; Hartwig, J. F.; Miyaura, N. Angew. Chem., Int. Ed. 2002, 41, 3056.

[46] Ishiyama, T.; Isou, H.; Kikuchi, T.; Miyaura, N. Chem. Commun. 2010, 46, 159

[47] Itoh, H.; Kikuchi, T.; Ishiyama, T.; Miyaura, N. Chem. Lett. 2011, 40, 1007.

[48] Sasaki, I.; Amou, T.; Ito, H.; Ishiyama, T. Org. Biomol. Chem. 2014, 12, 2041.

[49] Ros, A.; Estepa, B.; Lopez-Rodriguez, R.; Alvarez, E.; Fernández, R.; Lassaletta, J. M. Angew. Chem., Int. Ed. 2011, 50, 11724.

[50] Ros, A.; Lopez-Rodriguez, R.; Estepa, B.; Alvarez, E.; Fernández, R.; Lassaletta, J. M. J. Am. Chem. Soc. 2012, 134, 4573.

[51] Lopez-Rodriguez, R.; Ros, A.; Fernández, R.; Lassaletta, J. M. J. Org. Chem. 2012, 77, 9915.

[52] Roering, A. J.; Hale, L. V. A.; Squier, P. A.; Ringgold, M. A.; Wiederspan, E. R.; Clark, T. B. Org. Lett. 2012, 14, 3558.

[53] Hale, L. V. A.; McGarry, K. A.; Ringgold, M. A.; Clark, T. B. Organometallics 2014, 34, 51.

[54] Hale, L. V. A.; Emmerson, D. G.; Ling, E. F.; Roering, A. J.; Ringgold, M. A.; Clark, T. B. Org. Chem. Front. 2015, 2, 661.

[55] Roosen, P. C.; Kallepalli, V. A.; Chattopadhyay, B.; Singleton, D. A.; Maleczka, R. E.; Smith, M. R. J. Am. Chem. Soc. 2012, 134, 11350 .

[56] Bisht, R.; Chattopadhyay, B. J. Am. Chem. Soc. 2016, 138, 84

[57] Ghaffari, B.; Preshlock, S. M.; Plattner, D. L.; Staples, R. J.; Maligres, P. E.; Krska, S. W.; Maleczka, R. E., Jr.; Smith, M. R. J. Am. Chem. Soc. 2014, 136, 14345.

[58] Wang, G.; Liu, L.; Wang, H.; Ding, Y. S.; Zhou, J.; Mao, S.; Li, P. J. Am. Chem. Soc. 2017, 139, 91.

[59] Gorovoy, A. S.; Gozhina, O. V.; Svendsen, J. S.; Domorad, A. A.; Tetz, G. V.; Tetz, V. V.; Lejon, T. Chem. Biol. Drug Des. 2013, 81, 408.

[60] Andrés, P.; Ballano, G.; Calaza, M. I.; Cativiela, C. Chem. Soc. Rev. 2016, 45, 2291.

[61] Smoum, R.; Rubinstein, A.; Dembitsky, V. M.; Srebnik, M. Chem. Rev. 2012, 112, 4156.

[62] Wang, Y.-X.; Zhang, P.-F.; Ye, M. Chin. J. Chem. 2020, 38, 1762.

[63] Zhan, M.; Song, P.; Jiao, J.; Li, P. Chin. J. Chem. 2020, 38, 665.

[64] Zou, X.; Zhao, H.; Li, Y.; Gao, Q.; Ke, Z.; Xu, S. J. Am. Chem. Soc. 2019, 141,5334

[65] Yang, Y.; Gao, Q.; Xu, S. Adv. Syn. Catal. 2019, 361, 858.

[66] Kawamorita, S.; Ohmiya, H.; Hara, K.; Fukuoka, A.; Sawamura, M. J. Am. Chem. Soc. 2009, 131, 5058

[67] Kawamorita, S.; Ohmiya, H.; Sawamura, M. J. Org. Chem. 2010, 75,3855 . 
[68] Yamazaki, K.; Kawamorita, S.; Ohmiya, H.; Sawamura, M. Org. Lett. 2010, 12, 3978.

[69] Kawamorita, S.; Miyazaki, T.; Ohmiya, H.; Iwai, T.; Sawamura, M. J. Am. Chem. Soc. 2011, 133, 19310.

[70] Kawamorita, S.; Miyazaki, T.; Iwai, T.; Ohmiya, H.; Sawamura, M. J. Am. Chem. Soc. 2012, 134, 12924.

[71] Jiang, Q.; Duan-Mu, D.; Zhong, W.; Chen, H.; Yan, H. Chem.-Eur. J. 2013, 19, 1903.

[72] Miura, W.; Hirano, K.; Miura, M. Org. Lett. 2016, 18, 3742.

[73] Crawford, K. M.; Ramseyer, T. R.; Daley, C. J. A.; Clark, T. B. Angew. Chem., Int. Ed. 2014, 53, 7589.

[74] Guo, W. H.; Min, Q. Q.; Gu, J. W.; Zhang, X. Angew. Chem., Int. Ed. 2015, 54, 9075.

[75] Frémont, P.; Marion, N.; Nolan, S. P. Coord. Chem. Rev. 2009, 253, 862.

[76] Zhu, D.; Chen, L.; Fan, H.; Yao, Q.; Zhu, S. Chem. Soc. Rev. 2020, 49, 908.

[77] Keske, E. C.; Moore, B. D.; Zenkina, O. V.; Wang, R.; Schatte, G.; Crudden, C. M. Chem. Commun. 2014, 50, 9883.

[78] Zhong, L.; Zong, Z.-H.; Wang, X.-C. Tetrahedron 2019, 75, 2547.

[79] Queval, P.; Jahier, C.; Rouen, M.; Artur, I.; Legeay, J.-C.; Falivene, L.; Toupet, L.; Crévisy, C.; Cavallo, L.; Baslé, O.; Mauduit, M. Angew. Chem., Int. Ed. 2013, 52, 14103.

[80] Thongpaen, J.; E. Schmid, T.; Toupet, L.; Dorcet, V.; Mauduit, M.; Baslé, O. Chem. Commun. 2018, 54, 8202.

[81] Kainz, Q. M.; Matier, C. D.; Bartoszewicz, A.; Zultanski, S. L.; Peters, J. C.; Fu, G. C. Science 2016, 351, 681.

[82] Terrett, J. A.; Cuthbertson, J. D.; Shurtleff, V. W.; MacMillan, D. W. C. Nature 2015, 524, 330 .

[83] Tellis, J. C.; Primer, D. N.; Molander, G. A. Science 2014, 345, 433.

[84] Thongpaen, J.; Manguin, R.; Dorcet, V.; Vives, T.; Duhayon, C.;
Mauduit, M.; Baslé, O. Angew. Chem., Int. Ed. 2019, 58, 15244.

[85] Lyons, T. W.; Sanford, M. S. Chem. Rev. 2010, 110, 1147.

[86] He, J.; Wasa, M.; Chan, K. S. L.; Shao, Q.; Yu, J. Chem. Rev. 2017 117,8754 .

[87] Topczewski, J. J.; Sanford, M. S. Chem. Sci. 2015, 6, 70.

[88] Davan, T.; Corcoran, E. W.; Sneddon, L. G. Organometallics 1983, 2,1693

[89] Kadlecek, D. E.; Carroll, P. J.; Sneddon, L. G. J. Am. Chem. Soc. 2000, 122, 10868

[90] Tatsuo, I.; Kousaku, I.; Jun, T.; Norio, M. Chem. Lett. 2001, 30, 1082 .

[91] Ohmura, T.; Kijima, A.; Suginome, M. J. Am. Chem. Soc. 2009, 131,6070 .

[92] Dai, H. X.; Yu, J. Q. J. Am. Chem. Soc. 2012, 134, 134

[93] Kuninobu, Y.; Iwanaga, T.; Omura, T.; Takai, K. Angew. Chem., Int. Ed. 2013, 52, 4431.

[94] Yoshigoe, Y.; Kuninobu, Y. Org. Lett. 2017, 19, 3450.

[95] Arockiam, P. B.; Bruneau, C.; Dixneuf, P. H. Chem. Rev. 2012, 112, 5879.

[96] Ackermann, L. Acc. Chem. Res. 2014, 47, 281

[97] Wang, Z.; Xie, P.; Xia, Y. Chin. Chem. Lett. 2018, 29, 47.

[98] Fernández-Salas, J. A.; Manzini, S.; Piola, L.; Slawin, A. M.; Nolan, S. P. Chem. Commun. 2014, 50, 6782.

[99] Okada, S.; Namikoshi, T.; Watanabe, S.; Murata, M. ChemCatChem 2015, 7, 1531.

[100] Sarkar, S.; Kumar, N. Y.; Ackermann, L. Chem.-Eur. J. 2017, 23, 84.

[101] Maeda, Y.; Sato, M.; Okada, S.; Murata, M. Tetrahedron Lett. 2018 59, 2537.

[102] Yao, W.; Wang, J.; He, L.; Cao, D.; Yang, J. J. Org. Chem. 2020, 85,10245 . 